\title{
Maxwell field in spatially flat FLRW space-times
}

\author{
Ion I. Cotăescu ${ }^{\mathrm{a}}(1)$ \\ West University of Timişoara, V. Pârvan Ave. 4, 300223 Timisoara, Romania
}

Received: 16 August 2021 / Accepted: 28 September 2021 / Published online: 15 October 2021

(C) The Author(s) 2021

\begin{abstract}
The classical and quantum theory of the Maxwell free field (or perturbation) minimally coupled to the gravity of local-Minkowskian spatially flat Friedmann-LemaîtreRobertson-Walker (FLRW) space-times is constructed in conformal local charts (herein called frames) where the Maxwell equations have the same form as in special relativity. Taking into account that the conformal coordinates cannot be measured directly, all the obtained results are transformed in physical frames, with cosmic time and space coordinates of Painlevé type, where these may take on a physical meaning. In these frames, the Maxwell theory is equivalent to the electrodynamics in flat macroscopic media whose constitutive equations predict magnetoelectric type effects interpreted here as a geometric induction. The given example is of a system of static charges giving rise simultaneously to timedependent electric and magnetic fields that can be measured in physical frames. The quantization of the Maxwell free field in these manifolds is performed in a canonical manner using the momentum-helicity basis. The propagators in conformal and physical frames and the principal one-particle operators are written down. It is shown that this approach reveals a new behaviour of the one-particle wave packets during propagation and specific effects produced by the apparent horizons of the observers staying at rest in their proper physical frames.
\end{abstract}

\section{Introduction}

The Maxwell field holds a special position in physics as the only field having both classical and quantum behaviours. In general relativity, this field is involved in many models in astrophysics and cosmology, but it is less studied separately as a perturbation on semi-Riemannian manifolds. The general theory of the electromagnetic field on these manifolds is equivalent to the electrodynamics in flat macroscopic media where the permittivity and permeability are given by the com-

\footnotetext{
a e-mail: icotaescu@yahoo.com (corresponding author)
}

ponents of the metric tensor $[1,2]$. In this framework, the propagation of the electromagnetic field coupled to gravity was successfully studied using perturbations [2]. Moreover, exploiting this formalism and the conformal invariance of the Maxwell equations, analytical solutions of these equations were derived in co-moving frames with conformal time and spherical space coordinates of Friedmann-LemaitreRobertson-Walker (FLRW) space-times [3]. These solutions are spherical waves forming the total angular momentum basis in which the first canonical quantization of the Maxwell field on FLRW manifolds was performed deriving the oneparticle operators [3].

Other solutions of the Maxwell equations were studied in the de Sitter manifolds, either in local frames with static coordinates [4-8] or in co-moving frames of the expanding portions of arbitrary dimensions [9]. In the $(1+3)$-dimensional de Sitter expanding portion, known as the de Sitter expanding universe, we performed some time ago the canonical quantization of the Maxwell free field in the Coulomb gauge [10], which was the starting point in studying the de Sitter QED in the first order of perturbations [11], the quantum theory of redshift [12] and the propagation of the Maxwell wave packets in this manifold [13].

In these investigations, we exploited the conformal invariance of the Maxwell equations, allowing us to take over the results of the special relativistic QED in the conformal frames of the de Sitter expanding universe whose metrics are conformal transformations of the Minkowski one. However, such conformal frames can be introduced in any local Minkowskian spatially flat FLRW space-time, where we may generalize the results obtained in the de Sitter case as we intend to do in the present paper, proposing the classical and quantum theory of the Maxwell field in these FLRW manifolds.

The conformal coordinates of the co-moving frames, which help us to take over the results from the flat case are a very effective tool, but these are different from the measured physical ones which are formed by the proper (or cosmic) 
time and space coordinates of Painlevé type [14]. To avoid any ambiguity, we consider that the physical measurements can be done exclusively in the frames with physical coordinates. Therefore, our strategy is to use the conformal coordinates for taking over the known results from the flat case and then to transform them in the physical frames where these can be interpreted. Thus we may construct the classical theory of the Maxwell field in the spatially flat FLRW space-times.

With regard to the quantum approach, we must specify from the beginning that we adopt the standard interpretation of quantum mechanics [15]. We assume that the quantum states are prepared or measured by a global classical apparatus represented by the algebra of the vector fields, globally defined, which have to play the role of quantum observables. Of special interest are the Killing vector fields which are conserved observables commuting with the operators of the field equations. In this framework we may perform the canonical quantization of the Maxwell free field in conformal frames adopting the Coulomb gauge for eliminating the non-physical components as in special relativity $[16,17]$. The problem here is that the transformation to the physical frame depends on time changing, thus the time evolution picture [18-20]. To avoid this difficulty, we chose the initial time $t_{0}$ when the scale factor $a\left(t_{0}\right)=1$ and the physical and conformal space coordinates coincide. We show that this choice does not restrict the generality, since the conformal coordinates and the scale factor can be rescaled at any time without affecting the coordinates and geometry of the physical frames.

Technically speaking, we pay attention to a pair of sensitive problems which are crucial in our approach. The first one is related to the form of the plane-wave solutions of the Maxwell equations which must depend on the initial conditions, as the physical space coordinates are no longer homogeneous as the conformal ones. This dependence allows us to ensure the correct flat limits of these solutions, but only if we chose the initial time such that $a\left(t_{0}\right)=1$, which is just the mandatory condition for performing the canonical quantization. The second problem is related to the detector measuring propagating wave packets which must select only the radiation emitted by a remote source. To do so, we assume that the detector filters the photon momenta on a desired direction with the help of a suitable projection operator determining the amplitude of the detected one-dimensional wave packet and the expectation values and dispersions of the measured observables.

We start in the second section presenting the spatially flat FLRW geometry and the specific isometries, introducing the conformal and physical frames and showing how the conformal coordinates and the scale factor can be rescaled without affecting the physical ones. Moreover, we derive the null geodesics in terms of the conserved momentum and initial conditions [21], outlining possible effects due to the apparent horizon of an observer staying at rest in the origin of its proper frame with physical coordinates. To avoid further confusion, we consider the problem of two observers which emit and respectively detect a light beam measuring the kinetic parameters, including the peculiar momentum we defined recently [21]. This will be the general layout in which we study the classical or quantum effects during propagation.

The next section is devoted to the classical Maxwell field, whose solutions may be written in conformal frames, while the field strength and the energy-momentum tensor has to be measured in the associated physical frame. To achieve this, we first derive the transformation between these two frames, writing then the concrete expressions of the mentioned quantities in the physical frame. Here, we see in the equivalent electrodynamics in flat media that the constitutive equations have terms generating magnetoelectric type effects but which in fact are produced by the dynamics of the backgrounds. For this reason, here we use the term of geometric induction in physical frames, which must not be confused with the gravitational induction which addresses only the gravitational field (see, for instance, [22]). The example we give is a system of static charges giving rise simultaneously to a time-dependent Coulomb field associated with an induced magnetic one that can be observed in the physical frame.

The canonical quantization of the Maxwell field is presented in section 4. Following the method of Ref. [10], we start with the fundamental plane-wave solutions in the Coulomb gauge and conformal coordinates which are similar to those of special relativity but depending, in addition, on the initial conditions. These solutions form the momentumhelicity basis in which we perform the canonical quantization as in the de Sitter $[10,12]$ or the flat $[16,17]$ cases, deriving the commutator functions and propagators in conformal frames. These are two-point functions very similar to those of the flat case, depending only on the differences in the coordinates of these points. However, by transforming these functions in the physical frames, we obtain more complicated expressions, depending separately on the sets of coordinates of the two points. This indicates that the propagation in physical frames is quite different from that we encountered in the usual QED. This section ends by presenting the mode expansions in momentum representation of the principal one-particle operators.

In section 5 we study the propagation of the one-particle wave packets following the same steps as in the case of the de Sitter space-time [13]. Revisiting our problem of two observers, we assume that the first one prepares a 3dimensional wave packet which has to be detected by the second observer in its proper physical frame. We define the prepared wave packet in the conformal frame of the first observer and the projection operator which filters the onedimensional wave packet which has to be detected by the second observer. Then, transforming the observation data in 
the physical frame, we conclude that the detected wave packet propagates, accelerating from the initial velocity (less than the speed of light) up to the speed of light recorded by the detector. During propagation, this has an important decay and a moderate dispersion which are specific for the expanding backgrounds but absent in the flat case. We thus generalize the similar results we obtained in the de Sitter expanding universe [13].

Most interesting are the horizon effects we analyse in section 6 for the first time. We consider isotropic wave packets of the Cauchy-Lorentz type propagating in spatially flat FLRW expanding space-times with scale factors of the form $a(t) \propto t^{s}$ with $s>0$. In these geometries, the observers have, in their proper physical frames, dynamical apparent horizons expanding with constant velocities. We show how the space expansion drags back the wave packet which is starting slower. Moreover, if the packet is prepared at the initial moment outside the apparent horizon, where the dragging effect is stronger, then it starts in the backward direction decelerating and stopping when the horizon reaches it. Then this restarts in the forward direction accelerating up to the speed of light detected by the second observer. This new spectacular horizon effect cannot be observed directly but may be pointed out indirectly by measuring the redshift. Some comments on this matter and other concluding remarks are presented in the last section.

As we develop here both the classical and quantum approaches, we use natural Planck units with $c=\hbar=G=$ 1.

\section{Light in spatially flat FLRW space-times}

In local Minkowskian semi-Reiemannian space-times, $(M, g)$, the geodesic motion may be studied in frames $\{x\}$ with local coordinates $x^{\mu}$ (labelled by natural indices $\alpha, . ., \mu, v, \ldots=$ $0,1,2,3)$ and line elements

$d s^{2}=g_{\mu \nu}(x) \mathrm{d} x^{\mu} \mathrm{d} x^{\nu}$.

Integrating the geodesic equations, we introduce integration constants whose physical meaning may be better understood by relating them to the conserved quantities associated to the isometries of $M$ that preserve the metric. These transformations form the isometry group $I(M)$ whose elements $\mathfrak{g}(\xi)$ depend on the group parameters $\xi_{a}(\mathrm{a}, \mathrm{b}, \ldots=1,2, . . \mathrm{n})$. Each isometry,

$x^{\mu} \rightarrow x^{\prime \mu}=\phi_{\mathfrak{g}(\xi)}^{\mu}(x)=x^{\mu}+\left.\xi^{a} \partial_{\xi_{a}} \phi_{\mathfrak{g}(\xi)}^{\mu}(x)\right|_{\xi=0}+\cdots$,

defines the associated Killing vector $k_{a}$ having the components

$k_{a}^{\mu}(x)=\left.\partial_{\xi_{a}} \phi_{\mathfrak{g}(\xi)}^{\mu}(x)\right|_{\xi=0}$, which satisfy the Killing equation $k_{a \mu ; v}+k_{a v ; \mu}=0$. The Killing vectors generate the quantities

$\xi^{a} \rightarrow k_{a} \rightarrow K_{a}=k_{a \mu}(x) p^{\mu}, \quad p^{\mu}=\frac{\mathrm{d} x^{\mu}}{\mathrm{d} \lambda}$,

which are conserved along geodesics. For a particle of mass $m \geq 0$, the affine parameter $\lambda$ is defined as $\mathrm{d} s=m \mathrm{~d} \lambda$ such that the components $p^{\mu}$ of the four-momentum $p$ satisfy the condition $g_{\mu \nu}(x) p^{\mu} p^{\nu}=m^{2}$ resulting from Eq. (1).

\subsection{Frames and conserved quantities}

In what follows, we focus on the spatially flat FLRW spacetimes, denoted by $(M, a)$, where we consider two types of frames, namely the conformal pseudo-Euclidean frames $\left\{t_{c}, \mathbf{x}_{c}\right\}$ with conformal coordinates, $x_{c}^{\mu}$, and the physical frames $\{t, \mathbf{x}\}$ with physical coordinates, $x^{\mu}$, of the Painlevé type [14].

The conformal coordinates are the conformal time $t_{c}$ and the conformal Cartesian spaces coordinates $x_{c}^{i}(i, j, k, \ldots=$ $1,2,3)$, known as the co-moving space coordinates [23]. The line element

$\mathrm{d} s^{2}=g_{\mu \nu}^{c}\left(x_{c}\right) \mathrm{d} x_{c}^{\mu} \mathrm{d} x_{c}^{\nu}=a\left(t_{c}\right)^{2}\left(\mathrm{~d} t_{c}^{2}-\mathrm{d} \mathbf{x}_{c} \cdot d \mathbf{x}_{c}\right)$,

depends on the conformal scale factor $a\left(t_{c}\right)$. The corresponding metric tensors can be represented as

$g_{\mu \nu}^{c}=a\left(t_{c}\right)^{2} \eta_{\mu \nu}, \quad g^{c \mu \nu}=\frac{1}{a\left(t_{c}\right)^{2}} \eta_{\mu \nu}$,

where $\eta=\operatorname{diag}(1,-1,-1,-1)$ is the Minkowski metric. Then we may write

$\sqrt{g} \equiv \sqrt{-\operatorname{det}\left(g_{\mu \nu}\right)} \rightarrow \sqrt{g^{c}}=a\left(t_{c}\right)^{4}$.

Note that the conformal coordinates were introduced for the first time by Lemaître [24] in de Sitter's universe.

The physical coordinates are the proper, or cosmic, time $t$ and the Cartesian space coordinates $x^{i}$ defined as

$t=\int a\left(t_{c}\right) \mathrm{d} t_{c}, \quad \mathbf{x}=\mathbf{x}_{c} a\left(t_{c}\right)$

such that the inverse transformation reads

$t_{c}=\int \frac{\mathrm{d} t}{a(t)}, \quad \mathbf{x}_{c}=\frac{\mathbf{x}}{a(t)}, \quad a(t) \equiv a\left[t_{c}(t)\right]$,

where $a(t)$ is the usual FLRW scale factor [23]. The physical frame is the proper frame of an observer which stays at rest in origin measuring the physical coordinates whose line element reads

$$
\begin{aligned}
d s^{2} & =g_{\mu \nu}(x) \mathrm{d} x^{\mu} \mathrm{d} x^{\nu} \\
& =\left[1-\frac{\dot{a}(t)^{2}}{a(t)^{2}} \mathbf{x}^{2}\right] \mathrm{d} t^{2}+2 \frac{\dot{a}(t)}{a(t)} \mathbf{x} \cdot d \mathbf{x} \mathrm{d} t-d \mathbf{x} \cdot d \mathbf{x},
\end{aligned}
$$


where

$\frac{\dot{a}(t)}{a(t)}=\frac{1}{a(t)} \frac{\mathrm{d} a(t)}{\mathrm{d} t}=\frac{1}{a\left(t_{c}\right)^{2}} \frac{\mathrm{d} a\left(t_{c}\right)}{\mathrm{d} t_{c}}$

is the Hubble function for which we do not use a special notation. The metric defined by the line element (10) has covariant

$$
\left(g_{\mu \nu}\right)=\left(\begin{array}{cccc}
1-\frac{\dot{a}^{2}}{a^{2}} \mathbf{x} \cdot \mathbf{x} & \frac{\dot{a}}{a} x^{1} & \frac{\dot{a}}{a} x^{2} & \frac{\dot{a}}{a} x^{3} \\
\frac{a}{a} x^{1} & -1 & 0 & 0 \\
\frac{\dot{a}}{a} x^{2} & 0 & -1 & 0 \\
\frac{a}{a} x^{3} & 0 & 0 & -1
\end{array}\right)
$$

and contravariant

$$
\left(g^{\mu \nu}\right)=\left(\begin{array}{cccc}
1 & \frac{\dot{a}}{a} x^{1} & \frac{\dot{a}}{a} x^{2} & \frac{\dot{a}}{a} x^{3} \\
\frac{\dot{a}}{a} x^{1} & \frac{\dot{a}^{2}}{a^{2}}\left(x^{1}\right)^{2}-1 & \frac{\dot{a}^{2}}{a^{2}} x^{1} x^{2} & \frac{\dot{a}^{2}}{a^{2}} x^{1} x^{3} \\
\frac{\dot{a}}{a} x^{2} & \frac{\dot{a}^{2}}{a^{2}} x^{1} x^{2} & \frac{\dot{a}^{2}}{a^{2}}\left(x^{2}\right)^{2}-1 & \frac{\dot{a}^{2}}{a^{2}} x^{2} x^{3} \\
\frac{\dot{a}}{a} x^{3} & \frac{\dot{a}^{2}}{a^{2}} x^{1} x^{3} & \frac{\dot{a}^{2}}{a^{2}} x^{2} x^{3} & \frac{\dot{a}^{2}}{a^{2}}\left(x^{3}\right)^{2}-1
\end{array}\right)
$$

components such that $\sqrt{g}=1$. In this frame the observer has an apparent horizon which is a sphere of the time-dependent radius

$r_{a}(t)=\frac{a(t)}{\dot{a}(t)}$,

which restricts the space measurement at the time $t$ to the domain $|\mathbf{x}|<r_{a}(t)$. In general, this apparent horizon is not an event horizon, as this does not separate disjoint domains of causality.

Assuming that the physical measurement can be performed exclusively in the physical frames, we observe that the scaling

$t_{c} \rightarrow \sigma t_{c}, \quad \mathbf{x}_{c} \rightarrow \sigma \mathbf{x}_{c}, \quad a \rightarrow \frac{a}{\sigma}$

does not affect the physical coordinates and the Hubble function. This scaling will be useful when we discuss the role of the initial condition in determining some significant geometric quantities and applying the quantization procedure.

The spatially flat FLRW space-times have the isometry group $I(M)=E(3)$ formed by the space translations

$t_{c}^{\prime}=t_{c}, \quad t^{\prime}=t$,

$x_{c}^{\prime i}=x_{c}^{i}+\xi^{i}, \rightarrow x^{\prime i}=x^{i}+\xi^{i} a(t)$

and the global rotations

$t_{c}^{\prime}=t_{c}, \quad t^{\prime}=t$,

$x_{c}^{\prime i}=R_{j}^{i}(\omega) x_{c}^{j}, \quad \rightarrow x^{\prime i}=R_{j}^{i}(\omega) x^{j}$

where $R_{j}^{i}(\omega)=\delta_{j}^{i}-\omega_{j}^{i}+\cdots$. In the conformal frame, where the translations do not depend on time, we may derive the associated Killing vectors having the simpler components [21]

$$
\xi^{i} \rightarrow k_{(i)}: k_{(i)}^{0}=0, \quad k_{(i)}^{j}=\delta_{i}^{j},
$$

$$
\omega^{i j} \rightarrow k_{(i j)}: k_{(i j)}^{0}=0, \quad k_{(i j)}^{k}=\delta_{j}^{k} x_{c}^{i}-\delta_{i}^{k} x_{c}^{j} .
$$

These give rise to the conserved quantities

$P^{i}=-k_{(i)} j \frac{\mathrm{d} x_{c}^{j}}{\mathrm{~d} \lambda}=a\left(t_{c}\right)^{2} \frac{\mathrm{d} x_{c}^{i}}{\mathrm{~d} \lambda}$,

$L_{k}=-\frac{1}{2} \varepsilon_{k i j} k_{(i j) l} \frac{\mathrm{d} x_{c}^{l}}{\mathrm{~d} \lambda}=a\left(t_{c}\right)^{2} \varepsilon_{k i j} x_{c}^{i} \frac{\mathrm{d} x_{c}^{j}}{\mathrm{~d} \lambda}=\varepsilon_{k i j} x_{c}^{i} P^{j}$,

representing the components of the conserved momentum $\mathbf{P}=\mathbf{n}_{P} P(P=|\mathbf{P}|)$ and respectively the angular momentum $\mathbf{L}=\mathbf{x}_{c} \wedge \mathbf{P}$. Note that the indices $(i)$ and $(i j)$ are not natural ones, being in fact Euclidean indices, labelling the parameters of the $E(3)$ group whose upper or lower positions are equivalent.

In conformal frames, the geodesics of the particles of mass $m$ are determined by the initial condition $\mathbf{x}_{c 0}=\mathbf{x}\left(t_{c 0}\right)$ and the conserved momentum $\mathbf{P}$, which gives rise to the components of the covariant four-momentum as [21]

$p_{c}^{0}\left(t_{c}\right)=\frac{\mathrm{d} t_{c}}{\mathrm{~d} \lambda}=\frac{1}{a\left(t_{c}\right)^{2}} \sqrt{m^{2}+\frac{P^{2}}{a\left(t_{c}\right)^{2}}}$,

$p_{c}^{i}\left(t_{c}\right)=\frac{\mathrm{d} x_{c}^{i}}{\mathrm{~d} \lambda}=\frac{P^{i}}{a\left(t_{c}\right)^{2}}$

so that

$a\left(t_{c}\right)^{2}\left(p_{c}^{0^{2}}-\mathbf{p}_{c} \cdot \mathbf{p}_{c}\right)=m^{2}$.

The angular momentum is not explicitly involved in the geodesic equation, but can be related to the initial condition as $\mathbf{L}=\mathbf{x}_{c 0} \wedge \mathbf{P}$.

\subsection{Null geodesics}

The classical trajectories of light are the null geodesics (with $m=0$ ) whose kinetic quantities may be measured by an observer staying at rest in the origin $O$ of its proper frame with physical coordinates $\{t, \mathbf{x}\}_{O}$. These can be derived starting with the components

$k_{c}^{0}\left(t_{c}\right)=\frac{P}{a\left(t_{c}\right)^{2}}, \quad k_{c}^{i}\left(t_{c}\right)=\frac{P^{i}}{a\left(t_{c}\right)^{2}}$,

of the four-momentum in the conformal frame $\left\{t_{c}, \mathbf{x}_{c}\right\}_{O}$ of our observer, where the prime integral (24) becomes

$k_{c}^{0}\left(t_{c}\right)^{2}-\mathbf{k}_{c}\left(t_{c}\right)^{2}=0$.

In this frame, the null geodesics result simply by integrating the equation

$\frac{\mathrm{d} x_{c}^{i}}{\mathrm{~d} t_{c}}=\frac{k_{c}^{i}\left(t_{c}\right)}{k_{c}^{0}\left(t_{c}\right)}=n_{P}^{i}$

that yields the closed form

$\mathbf{x}_{c}\left(t_{c}\right)=\mathbf{x}_{c 0}+\mathbf{n}_{P}\left(t_{c}-t_{c 0}\right)$ 
which holds in any spatially flat FLRW space-time $[21,25]$.

The corresponding physical quantities measured by the observer $O$ in its proper frame, $\{t, \mathbf{x}\}_{O}$, may be obtained by substituting the physical coordinates according to Eq. (9). Thus we find the covariant components [21],

$k^{0}(t)=\frac{\mathrm{d} t}{\mathrm{~d} \lambda}=\frac{P}{a(t)}$,

$k^{i}(t)=\frac{\mathrm{d} x^{i}}{\mathrm{~d} \lambda}=\frac{P^{i}}{a(t)}+x^{i}(t) \frac{\dot{a}(t)}{a(t)} k^{0}(t)$,

which represent the measured energy and covariant momentum in the point $[t, \mathbf{x}(t)]$ of the null geodesic,

$\mathbf{x}(t)=\mathbf{x}_{0} \frac{a(t)}{a\left(t_{0}\right)}+\mathbf{n}_{P} a(t)\left[t_{c}(t)-t_{c}\left(t_{0}\right)\right]$,

which is passing through the space point $\mathbf{x}\left(t_{0}\right)=\mathbf{x}_{0}$ at the initial time $t_{0}$.

We recently showed that the covariant momentum defined by Eq. (30) can be split as $\mathbf{k}(t)=\hat{\mathbf{k}}(t)+\overline{\mathbf{k}}(t)$, where

$\hat{\mathbf{k}}(t)=\frac{\mathbf{P}}{a(t)}$,

$\overline{\mathbf{k}}=\mathbf{x}(t) \frac{\dot{a}(t)}{a(t)} k^{0}(t)$,

are the peculiar and recessional momenta, respectively [21]. We must stress that this splitting can be done only in the physical frames where the prime integral derived from the line element (10) gives the familiar identity

$k^{0}(t)^{2}-\hat{\mathbf{k}}(t) \cdot \hat{\mathbf{k}}(t)=0$,

which is just the mass-shell condition of special relativity satisfied by the energy and peculiar momentum. Therefore, we may conclude that the peculiar momentum produces the entire energy of the geodesic motion as in special relativity.

In the physical frame, the conserved angular momentum is related on the initial condition as

$$
\begin{aligned}
\mathbf{L} & =\mathbf{x}(t) \wedge \mathbf{k}(t)=\mathbf{x}(t) \wedge \hat{\mathbf{k}}(t) \\
& =\frac{\mathbf{x}(t) \wedge \mathbf{P}}{a(t)}=\frac{\mathbf{x}_{0} \wedge \mathbf{P}}{a\left(t_{0}\right)} .
\end{aligned}
$$

Obviously, this vanishes when the observer $O$ stays at rest in a space point of the measured geodesic.

\subsection{Two observers problem}

The recessional momentum (33) depends explicitly on coordinates such that the experimental results will depend on the relative position between the detector and source of the measured particle. This is not an impediment, as the peculiar and recessional contributions can be separated at any time without ambiguities. Nevertheless, to avoid confusion, we take care on this dependence, looking for suitable positions of the observer's proper frames in order to obtain intuitive results.
For this purpose, we take over the common layout of Refs. $[12,13,21]$, in which two observers measure the motion of a photon on a null geodesic which is passing through the origins $O$ and $O^{\prime}$ of their proper frames, $\{t, \mathbf{x}\}_{O}$ and $\left\{t, \mathbf{x}^{\prime}\right\}_{O^{\prime}}$, respectively. We assume that at the initial time $t_{0}$, the origin $O^{\prime}$ is translated with respect to $O$ as

$\mathbf{x}^{\prime}\left(t_{0}\right)=\mathbf{x}\left(t_{0}\right)+\mathbf{d}\left(t_{0}\right)$,

where $\mathbf{d}(t)=\mathbf{d} a(t)$ depends on the translation parameters of Eq. (16) denoted now by $\xi^{i}=d^{i}$. Then it is convenient to introduce the unit vector $\mathbf{n}$ of the direction $O^{\prime} O$ such that $\mathbf{d}=\mathbf{n} d$. Now we find that the velocity of $O^{\prime}$ with respect to $O, \mathbf{v}(t)=-\dot{\mathbf{d}}(t)=-\frac{\dot{a}(t)}{a(t)} \mathbf{d}(t)$, complies with the velocity law, $v=a(t) d$, that is sometimes confused with the Hubble one [29].

Our experiment starts in this layout at the time $t_{0}$ when the observer $O^{\prime}$ emits a photon of momentum $\mathbf{k}=\mathbf{n} k$ and energy $k$ on the null geodesic $O^{\prime} \rightarrow O$ that can be written as

$x(t)=a(t)\left[t_{c}(t)-t_{c}\left(t_{0}\right)-d\right]$,

where $x(t)=\mathbf{n} \cdot \mathbf{x}(t)$. We look first for the energy and momentum of this photon measured in the origin $O$ at the final time $t_{f}$, when the photon is detected. For solving this problem, we start with the conserved momentum observed in $O^{\prime}$,

$\mathbf{P}^{\prime}=\mathbf{P}=\mathbf{n}_{P} P=\mathbf{k} a\left(t_{0}\right)$,

denoting $P=k a\left(t_{0}\right)$ and $\mathbf{n}_{P}=\mathbf{n}$. Then, by substituting it in Eqs. (29) and (30), we obtain the energy and momentum in $O$,

$k^{0}\left(t_{f}\right)=k \frac{a\left(t_{0}\right)}{a\left(t_{f}\right)}$,

$$
\mathbf{k}\left(t_{f}\right)=\hat{\mathbf{k}}\left(t_{f}\right)=-\mathbf{n} k \frac{a\left(t_{0}\right)}{a\left(t_{f}\right)},
$$

where $t_{f}$ satisfies the condition $x\left(t_{f}\right)=0$ which, according to Eq. (28), gives the simple algebraic equation

$t_{c}\left(t_{f}\right)=t_{c}\left(t_{0}\right)+d \rightarrow \int_{t_{0}}^{t_{f}} \frac{\mathrm{d} t}{a(t)}=d$,

which can be solved obtaining the value of $t_{f}$. Once we have this value, we can calculate the propagation time $t_{f}-t_{0}$ and the distance $d\left(t_{f}\right)$ between $O$ and $O^{\prime}$ at the time $t_{f}$,

$d\left(t_{f}\right)=d a\left(t_{f}\right)=d\left(t_{0}\right) \frac{a\left(t_{f}\right)}{a\left(t_{0}\right)}$,

thus completing the collection of the kinetic quantities related to this problem.

On the other hand, Eq. (39) shows that in expanding universes, a part of energy is lost during the propagation. This 
phenomenon gives rise to the redshift for which we recover the Lemaitre equation [26,27] of Hubble's law [28] as

$1+z=\frac{p^{0}\left(t_{0}\right)}{p^{0}\left(t_{f}\right)}=\frac{a\left(t_{f}\right)}{a\left(t_{0}\right)}$,

where $z$ is the usual redshift defined as the relative dilation of the wavelength [29].

In the proper physical frame of $O$, the velocity of the photon on the geodesic $O^{\prime} O$ can be derived from Eq. (37) as

$v(t)=\dot{x}(t)=1+\frac{\dot{a}(t)}{a(t)} x(t)$,

finding that the direction of the initial velocity,

$v\left(t_{0}\right)=1-\frac{\dot{a}\left(t_{0}\right)}{a\left(t_{0}\right)} d\left(t_{0}\right)=\left\{\begin{array}{l}>0 \text { if } d\left(t_{0}\right)<r_{a}\left(t_{0}\right) \\ =0 \text { if } d\left(t_{0}\right)=r_{a}\left(t_{0}\right), \\ <0 \text { if } d\left(t_{0}\right)>r_{a}\left(t_{0}\right)\end{array}\right.$,

depends on the position of $O^{\prime}$ with respect to the apparent horizon at the initial time $t_{0}$. The photon observed by $O$ is accelerated in the forward direction, such that the velocity is increasing from the initial value $v\left(t_{0}\right)$ up to the speed of light $v\left(t_{f}\right)=1$ detected by $O$ in $x\left(t_{f}\right)=0$. When $v\left(t_{0}\right)<$ 0 , then the photon starts in the backward direction, being decelerated until it stops and turns back, accelerating and moving straightforward to $O$. Note that for the observer $O^{\prime}$, the velocity of the emitted photon is always the speed of light, since from its point of view $d^{\prime}=0$.

Thus we outlined an important effect due to the dynamical apparent horizon that may interfere with the effects produced by the event horizons. As we intend to pay special attention to these effects, we need to know the ideal event horizon of an observer $O$ in its proper frame $\{t, \mathbf{x}\}_{O}$ in $(M, a)$. According to Rindler's definition [30,31], this is the distance

$r_{e}(t)=\int_{t}^{\infty} \mathrm{d} t^{\prime} \frac{a(t)}{a\left(t^{\prime}\right)}$,

for which the photon emitted by $O^{\prime}$ at the time $t$ never arrives in $O$ as $t_{f} \rightarrow \infty$ in Eq. (41). The examples analysed so far show that, at least in the expanding space-times of interest in cosmology, the event horizon is larger than the apparent one. The exception is the de Sittter expanding universe having the scale factor $a(t)=\exp \left(\omega_{H} t\right)$, where $\omega_{H}$ is the de Sitter-Hubble constant in our notation. This gives rise to time-independent overlapping horizons on the sphere of radius $r_{e}=r_{a}=\omega_{H}^{-1}$.

\section{Classical Maxwell field}

The classical theory of the Maxwell field is a Lagrangian theory in which the electromagnetic potential plays the role of canonical variable. The advantage of this approach is deriving the conserved quantities associated to isometries via Noether's theorem. Let us start by briefly revisiting this procedure, which has to be applied to the $E(3)$ isometries of the space-times $(M, a)$.

\subsection{General framework}

In any space-time, $(M, g)$, the electromagnetic potential $A$, minimally coupled to the gravity of background, has the action

$\mathcal{S}[A]=\int \mathrm{d}^{4} x \sqrt{g}\left[\mathcal{L}(A)-A_{\mu} J^{\mu}\right]$,

where $\sqrt{g}$ is defined by Eq. (7), while the Lagrangian of the free electromagnetic field

$\mathcal{L}(A)=-\frac{1}{4} F_{\mu \nu} F^{\mu \nu}$,

is expressed in terms of the field strength

$F_{\mu \nu}=\partial_{\mu} A_{\nu}-\partial_{\nu} A_{\mu}$.

The current density $J$ is conserved, $\partial_{\mu}\left(\sqrt{g} J^{\mu}\right)=0$, being the source of the field equations

$\partial_{\nu}\left(\sqrt{g} g^{\nu \alpha} g^{\mu \beta} F_{\alpha \beta}\right)=\sqrt{g} J^{\mu}$

of the classical Maxwell field in $(M, g)$.

For $J=0$, the theory of free field is invariant under gauge transformations, $A_{\mu} \rightarrow A_{\mu}+\partial_{\mu} \alpha$, and the conformal ones that transform simultaneously the metric tensor, $g_{\mu \nu} \rightarrow g_{\mu \nu}^{\prime}=\Omega g_{\mu \nu}$, and the potential components, $A_{\mu} \rightarrow A_{\mu}^{\prime}=A_{\mu}$ and $A^{\mu} \rightarrow A^{\prime \mu}=\Omega^{-1} A^{\mu}$. In addition, the canonical variables $A_{\mu}$ must comply with the Lorenz condition

$\partial_{\mu}\left(\sqrt{g} g^{\mu v} A_{\nu}\right)=0$,

which is no longer conformally invariant since

$\partial_{\mu}\left(\sqrt{g^{\prime}} g^{\prime \mu v} A_{v}^{\prime}\right)=\Omega \partial_{\mu}\left(\sqrt{g} g^{\mu v} A_{\nu}\right)+\sqrt{g} A^{\mu} \partial_{\mu} \Omega$.

In general, we can overcome this inconvenience by fixing a convenient gauge.

If $(M, g)$ allows isometries, then the vector field $A$ transforms under an isometry (2) as $A \rightarrow A^{\prime}=T_{\xi} A$, according to the operator-valued representation $\xi \rightarrow T_{\xi}$ of the isometry group $I(M)$ defined by the well-known rule

$\frac{\partial \phi_{\xi}^{v}(x)}{\partial x_{\mu}}\left(T_{\xi} A\right)_{\nu}[\phi(x)]=A_{\mu}(x)$

of the vector representation. The corresponding basis generators, $X_{a}=\left.i \partial_{\xi_{a}} T_{\xi}\right|_{\xi=0}$, have the action

$k_{a} \rightarrow\left(X_{a} A\right)_{\mu}=-i\left[k_{a}^{v} A_{\mu ; \nu}+k_{a ; \mu}^{v} A_{\nu}\right]$,

where $k_{a}$ are the Killing vectors (3) associated to the parameters $\xi^{a}$. These generators are differential operators that form the basis of the vector representation of the Lie algebra $i(M)$ 
of the group $I(M)$. Then, the Noether theorem provides us with the conserved quantities

$\xi^{a} \rightarrow k_{a} \rightarrow C_{a}=-\int_{\Sigma} \mathrm{d} \sigma_{\mu} \sqrt{g} T^{\mu \nu} k_{a \nu}$,

where

$T^{\mu \nu}=F^{\mu \alpha} F_{\alpha \cdot}^{\cdot v}-g^{\mu \nu} \mathcal{L}$

is the stress-energy tensor of the electromagnetic field while the integral is performed on the space-like section $\Sigma$ of $M$.

The theory of the Maxwell field on $(M, g)$ can be interpreted formally in terms of the electrodynamics of macroscopic media in flat space-times [1,2] involving the field's strength components

$$
\begin{aligned}
E_{i} & =F_{i 0}, & B_{i} & =\frac{1}{2} \varepsilon_{i j k} F_{j k}, \\
D_{i} & =\sqrt{g} F^{0 i}, & H_{i} & =\sqrt{g} \frac{1}{2} \varepsilon_{i j k} F^{j k},
\end{aligned}
$$

related through the constitutive equations [2]

$D_{i}=E_{i}+\varepsilon_{i j} E_{j}+\varepsilon_{i j k} g_{j} H_{k}$,

$B_{i}=H_{i}+\varepsilon_{i j} H_{j}-\varepsilon_{i j k} g_{j} E_{k}$,

where

$\varepsilon_{i j}=-\delta_{i j}-\sqrt{g} \frac{g^{i j}}{g_{00}}, \quad g_{j}=\frac{g_{j 0}}{g_{00}}$.

In metrics where $g_{i 0} \neq 0$, the last terms of the constitutive equations play the role of magnetoelectric susceptibilities, giving rise to magnetoelectric-type effects but which are produced by the background structure and dynamics.

In this formalism, the stress-energy tensor may be written in traditional manner as [2]

$$
\begin{aligned}
& \sqrt{g} T_{\cdot 0}^{0 \cdot}=\frac{1}{2}(\mathbf{E} \cdot \mathbf{D}+\mathbf{H} \cdot \mathbf{B}) \\
& \sqrt{g} T_{\cdot i}^{0 \cdot}=\varepsilon_{i j k} D_{j} B_{k}, \\
& \sqrt{g} T_{\cdot \cdot \cdot}^{i}=\varepsilon_{i j k} E_{j} H_{k}, \\
& \sqrt{g} T_{\cdot j}^{i \cdot}=D_{i} E_{j}+B_{i} H_{j}-\frac{1}{2} \delta_{i j}(\mathbf{D} \cdot \mathbf{E}+\mathbf{B} \cdot \mathbf{H}),
\end{aligned}
$$

thus completing the analogy with the electrodynamics of flat macroscopic media.

\subsection{Maxwell field in conformal and physical frames}

In the space-times $(M, a)$, the Maxwell field can be written down in conformal frames where the electromagnetic potential $A^{c}$ satisfies the Maxwell equations (50) which have the same form as in Minkowski space-time,

$\partial_{\mu}\left(\eta^{\mu \alpha} \eta^{\nu \beta}\left(\partial_{\alpha} A_{\beta}^{c}-\partial_{\beta} A_{\alpha}^{c}\right)\right)=\sqrt{g^{c}} J_{c}^{\nu}$,

because of their invariance under conformal transformations.
It is convenient to write down the equations of the free field (with $J=0$ ) in the familiar form

$\begin{aligned}-\Delta_{c} A_{0}^{c}+\partial_{t_{c}}\left(\partial_{x_{c}^{J}} A_{j}^{c}\right) & =0, \\ \partial_{t_{c}}^{2} A_{i}^{c}-\Delta_{c} A_{i}^{c}-\partial_{x_{c}^{i}}\left(\partial_{t_{c}} A_{0}^{c}-\partial_{x_{c}^{j}} A_{j}^{c}\right) & =0,\end{aligned}$

observing that the Lorenz condition (51), which is no longer conformally invariant, takes the form

$$
\begin{aligned}
0 & =\frac{1}{a\left(t_{c}\right)^{2}} \partial_{\mu}\left(a\left(t_{c}\right)^{2} \eta^{\mu v} A_{v}^{c}\right) \\
& =\partial_{t_{c}} A_{0}^{c}-\partial_{x_{c}^{i}} A_{i}^{c}+2 \frac{\dot{a}\left(t_{c}\right)}{a\left(t_{c}\right)} A_{0}^{c} .
\end{aligned}
$$

This lays out the last term that can be removed only in the Coulomb gauge

$A_{0}^{c}=0, \quad \partial_{x_{c}^{i}} A_{i}^{c}=0$,

when the system (64) and (65) reduces to the d'Alambert equation

$$
\left(\partial_{t_{c}}^{2}-\Delta_{c}\right) \mathbf{A}=0 \text {. }
$$

In any other gauge, including the Lorenz one, the contribution of this new term must be evaluated analysing concrete examples. The solutions of Eq. (68) give rise to the field strength which has the components

$F_{c \mu \nu}=\partial_{\mu} A_{\nu}^{c}-\partial_{\nu} A_{\mu}^{c}, \quad F_{c}^{\mu \nu}=\frac{1}{a\left(t_{c}\right)^{4}} \eta^{\mu \alpha} \eta^{\nu \beta} F_{c \alpha \beta}$,

leading to trivial constitutive equations, $D_{i}^{c}=E_{i}^{c}$ and $B_{i}^{c}=H_{i}^{c}$. These components as well as those of the corresponding stress-energy tensor $T_{c}$, defined by Eq. (56), are not measurable quantities, but help us to simply derive the physical quantities measured in physical frames.

We have seen that the space-times $(M, a)$ have $E(3)$ isometries generated by operators acting as in Eq. (54). The basis generators which are interpreted as the momentum and total angular momentum operators act as

$$
\begin{aligned}
k_{(i)} \rightarrow \hat{P}^{i} A_{j}^{c} & =-i \frac{\partial}{\partial x_{c}^{i}} A_{j}^{c}, \\
\varepsilon_{i j k} k_{(j k)} & \rightarrow \hat{J}_{i} A_{j}^{c}=\hat{L}_{i} A_{j}^{c}-i \varepsilon_{i j k} A_{k}^{c},
\end{aligned}
$$

where $\hat{\mathbf{L}}=\mathbf{x}_{c} \times \hat{\mathbf{P}}$ is the usual angular momentum operator. In addition, we define the Pauli-Lubanski (or helicity) operator $\hat{W}=\hat{\mathbf{P}} \cdot \hat{\mathbf{J}}$ whose action depends only on the spin parts,

$\hat{W} A_{i}^{c}=\varepsilon_{i j k} \frac{\partial}{\partial x_{c}^{j}} A_{k}^{c}$.

This operator will define the polarization in the canonical basis of the so(3) algebra as in special relativity [10]. Then we observe that in the conformal frame and Coulomb gauge, 
the conserved quantities (55) can be derived with the help of the identity

$$
\begin{aligned}
k_{a} \rightarrow C_{a} & =-\int \mathrm{d}^{3} x_{c} a\left(t_{c}\right)^{4} T_{c}^{0 v} k_{a v} \\
& =-\frac{1}{2} \eta^{\mu \nu}\left(A_{\mu}^{c},\left(X_{a} A^{c}\right)_{v}\right),
\end{aligned}
$$

involving the Hermitian form

$$
(f, g)=i \int \mathrm{d}^{3} x_{c} f^{*}\left(t_{c}, \mathbf{x}_{c}\right) \stackrel{\leftrightarrow}{\partial_{t_{c}}} g\left(t_{c}, \mathbf{x}_{c}\right)
$$

written with the notation $f \stackrel{\leftrightarrow}{\partial} g=f \partial g-g \partial f$. Now, by substituting $X_{a}$ by the operators defined by Eqs. (70), (71) and (72), we obtain all the conserved quantities we need.

The physical field strength and stress energy tensor cannot be derived directly in the physical frames where the Maxwell equations are complicated because of the non-diagonal metric tensor (12). Therefore, we are forced to solve the Maxwell equation in conformal frames, transforming then the final results into the physical ones. This can be done by transforming the potential $A^{c}$ derived in the conformal frame $\left\{t_{c}, \mathbf{x}_{c}\right\}_{O}$ into the potential $A$ of the physical frame $\{t, \mathbf{x}\}_{O}$, of the same observer, performing the substitution (9) as

$$
\begin{aligned}
A_{0}(t, \mathbf{x})= & A_{\mu}^{c} \frac{\partial x_{c}^{\mu}}{\partial t}=\frac{1}{a(t)} A_{0}^{c}\left(t_{c(t)}, \frac{x}{a(t)}\right) \\
& -\frac{\dot{a}(t)}{a(t)^{2}} x^{i} A_{i}^{c}\left(t_{c(t)}, \frac{x}{a(t)}\right), \\
A_{j}(t, \mathbf{x})= & A_{\mu}^{c} \frac{\partial x_{c}^{\mu}}{\partial x^{j}}=\frac{1}{a(t)} A_{j}^{c}\left(t_{c(t)}, \frac{x}{a(t)}\right) .
\end{aligned}
$$

These new potentials satisfy the Maxwell equation in the physical frame, giving covariant field strength components of the form (49), while the corresponding contravariant components take the form

$$
\begin{aligned}
F^{0 i}= & -F_{0 i}+\frac{\dot{a}}{a} F_{i j} x^{j}, \\
F^{i j}= & F_{i j}\left[1-\frac{\dot{a}^{2}}{a^{2}}\left(\left(x^{i}\right)^{2}+\left(x^{j}\right)^{2}\right)\right] \\
& +\frac{\dot{a}^{2}}{a^{2}}\left(x^{i} F_{j k}-x^{j} F_{i k}\right) x^{k}+\frac{\dot{a}}{a}\left(F_{0 i} x^{j}-F_{0 j} x^{i}\right) .
\end{aligned}
$$

These equations are equivalent to the constitutive equations (59) and (60), where now

$$
\varepsilon_{i j}=-\frac{\dot{a}^{2}}{a^{2}} \frac{x^{i} x^{j}}{1-\frac{\dot{a}^{2}}{a^{2}} \mathbf{x} \cdot \mathbf{x}}, \quad g_{i}=\frac{\dot{a}}{a} \frac{x^{i}}{1-\frac{\dot{a}^{2}}{a^{2}} \mathbf{x} \cdot \mathbf{x}},
$$

resulted from the metric tensor (12) (with $\sqrt{g}=1$ ). The stress-energy tensor can be written in compact form (62) or in terms of covariant $\left(E_{i}, B_{i}\right)$ or contravariant components $\left(D_{i}, H_{i}\right)$ by using Eqs. (59), (60) and (79). Thus we obtain all the quantities with physical meaning without solving the Maxwell equations in physical frames.

\subsection{Geometric induction}

We observe that Eq. (78) suggests a possible geometric induction of the electromagnetic field that may not be confused with the gravitational induction that addresses only the gravitational field [22]. In terms of the equivalent electrodynamics in flat media, this is a magnetoelectric effect produced by the electric field of components $E_{i}=F_{i 0}$ when $F_{i j}=0 \rightarrow B_{i}=0$. Then, from Eq. (78) we deduce that the background expansion induces the magnetic field

$\mathbf{H}=\frac{\dot{a}(t)}{a(t)} \mathbf{x} \wedge \mathbf{E}$,

proportional to the Hubble function. Conversely, if $F_{0 j}=0$, then the components $F_{i j}$ generate both the electric and magnetic fields according to Eqs. (77) and (78). We conclude that in the space-times $(M, a)$, the electric and magnetic fields cannot be separated as in the electrostatic and magnetostatic of the special relativistic electrodynamics.

The simplest example is the magnetic field of a static system of electric charges that is induced gravitationally by the evolution of the space-time $(M, a)$. Let us consider an observer $O$ which measures at the time $t_{0}$, when $a\left(t_{0}\right)=1$, a system of static charges of density $\rho(\mathbf{x})$ in its proper physical frame, $\{t, \mathbf{x}\}_{O}$. Then, bearing in mind that at the time $t_{0}$ the coordinates of the conformal and physical spaces coincide, we may write the current density in the conformal frame of the same observer as

$J_{c}^{0}=\frac{1}{\sqrt{g^{c}}} \rho\left(\mathbf{x}_{c}\right), \quad J_{c}^{i}=0$.

Introducing this current density in Eq. (63), we obtain the equation of the Coulomb potential

$-\Delta_{c} A_{0}^{c}\left(\mathbf{x}_{c}\right)=\rho\left(\mathbf{x}_{c}\right)$,

which is the same as in the flat case. Thus, we may write the well-known solution

$A_{0}^{c}\left(\mathbf{x}_{c}\right)=\frac{1}{4 \pi} \int \mathrm{d}^{3} x_{c}^{\prime} \frac{\rho\left(\mathbf{x}_{c}^{\prime}\right)}{\left|\mathbf{x}_{c}-\mathbf{x}_{c}^{\prime}\right|}$,

that holds in the conformal frame. Furthermore, we derive the scalar potential in the physical frame of $O$, resorting to the transformation (75) that yields

$A_{0}(t, \mathbf{x})=\frac{1}{4 \pi} \int \mathrm{d}^{3} x^{\prime} \frac{\rho\left(\mathbf{x}^{\prime}\right)}{\left|\mathbf{x}-a(t) \mathbf{x}^{\prime}\right|}$,

where $\mathbf{x}^{\prime}=\mathbf{x}_{c}^{\prime}$ is the original variable of integration at the time $t_{0}$. 
The field strength derived from Eqs. (77) and (78) is then formed by the electric field

$$
\mathbf{E}(t, \mathbf{x})=-\nabla A_{0}(t, \mathbf{x})=\frac{1}{4 \pi} \int \mathrm{d}^{3} x^{\prime} \rho\left(\mathbf{x}^{\prime}\right) \frac{\mathbf{x}-a(t) \mathbf{x}^{\prime}}{\left|\mathbf{x}-a(t) \mathbf{x}^{\prime}\right|^{3}},
$$

and the induced magnetic field (80) that now reads

$$
\mathbf{H}(t, \mathbf{x})=-\frac{\dot{a}(t)}{4 \pi} \int \mathrm{d}^{3} x^{\prime} \rho\left(\mathbf{x}^{\prime}\right) \frac{\mathbf{x} \wedge \mathbf{x}^{\prime}}{\left|\mathbf{x}-a(t) \mathbf{x}^{\prime}\right|^{3}} .
$$

The conclusion is that a static system of electric charges in a space-time $(M, a)$ produces time-dependent electric and magnetic fields simultaneously. In terms of the equivalent electrodynamics in flat media, this is a magnetoelectric effect due to the non-diagonal metric of the physical frames giving rise to a magnetoelectric susceptibility.

\section{Quantum Maxwell field}

The specific feature of the second quantization in general relativity is that for the massive fields on some backgrounds, such as the de Sitter expanding universe, one can define many vacua that may be involved in the mechanisms of the cosmological particle creation [23]. Fortunately, in the conformal frames of the FLRW space-times, one can take over from special relativity the method of canonical quantization of the Maxwell field, ensuring the uniqueness of the vacuum state. Thus, the first canonical quantization of this field was performed in the co-moving frames with spherical coordinates of the FLRW space-times by using the total angular momentum basis [3]. However, in spatially flat space-times $(M, a)$ we have the opportunity to use the momentum-helicity basis of the Maxwell field in Coulomb gauge [16,17], where the non-physical components of the electromagnetic potential are eliminated naturally without resorting to special artifices such as the Gupta-Blauler one [32,33]. In this gauge we performed the canonical quantization of the Maxwell field in the de Sitter expanding universe [10], postulating the commutation relations in the momentum-helicity basis. Here we would like to generalize this procedure to the space-times $(M, a)$, outlining the new specific features.

\subsection{Momentum-helicity basis}

In the conformal frame $\left\{t_{c}, \mathbf{x}_{\mathbf{c}}\right\}_{O}$ of the observer $O$, the potential $A^{c}$ in the Coulomb gauge (67) is a solutions of Eq. (68). Bearing in mind that in the associated physical frame $\{t, \mathbf{x}\}_{O}$ the space coordinates are no longer homogeneous as in the flat case, we need to specify where and when the solution is created. Therefore, we explicitly introduce the initial condi- tions $x_{c 0}=\left(t_{c 0}, \mathbf{x}_{c 0}\right)$ in the form of these solutions,

$$
\begin{aligned}
A_{i}^{c}\left(x_{c}-x_{c 0}\right)= & A_{i}^{c(+)}\left(x_{c}-x_{c 0}\right)+A_{i}^{c(-)}\left(x_{c}-x_{c 0}\right) \\
= & \int \mathrm{d}^{3} k \sum_{\lambda}\left[e_{i}\left(\mathbf{n}_{k}, \lambda\right) \hat{f}_{\mathbf{k}}\left(x_{c}-x_{c 0}\right) \hat{a}(\mathbf{k}, \lambda)\right. \\
& \left.+\left[e_{i}\left(\mathbf{n}_{k}, \lambda\right) \hat{f}_{\mathbf{k}}\left(x_{c}-x_{c 0}\right)\right]^{*} \hat{a}^{*}(\mathbf{k}, \lambda)\right], \quad(87)
\end{aligned}
$$

expressed in terms of wave functions in momentum representation, $\hat{a}(\mathbf{k}, \lambda)$, polarization vectors, $e_{i}\left(\mathbf{n}_{k}, \lambda\right)$, and fundamental solutions,

$\hat{f}_{\mathbf{k}}\left(x_{c}-x_{c 0}\right)=\frac{1}{(2 \pi)^{3 / 2}} \frac{1}{\sqrt{2 k}} e^{-i k\left(t_{c}-t_{c 0}\right)+i \mathbf{k} \cdot\left(\mathbf{x}_{c}-\mathbf{x}_{c 0}\right)}$,

where $\mathbf{k}=k \mathbf{n}_{k}$ is the momentum vector, with $k=|\mathbf{k}|$. What is new here is the phase

$\delta(\mathbf{k})=k t_{c 0}-\mathbf{k} \cdot \mathbf{x}_{c 0}$,

encapsulating the initial condition, which is neglected in the Minkowski space-time because of its homogeneity, i.e. the invariance under time and space translations. We must specify that in the space-times $(M, a)$, the initial condition has physical consequences, as we have shown recently in the case of the de Sitter expanding universe [12].

Another advantage of explicitly introducing the initial condition is that we can study how the flat limit can be reached correctly. Indeed, by writing the phase of the function (88),

$$
\begin{aligned}
& -k\left(t_{c}-t_{c 0}\right)+\mathbf{k} \cdot\left(\mathbf{x}_{c}-\mathbf{x}_{c 0}\right) \\
& =-k \int_{t_{0}}^{t} \frac{\mathrm{d} t}{a(t)}+\mathbf{k} \cdot\left[\frac{x}{a(t)}-\frac{x_{0}}{a\left(t_{0}\right)}\right] \\
& =\frac{1}{a\left(t_{0}\right)}\left[-k\left(t-t_{0}\right)+\mathbf{k} \cdot\left(\mathbf{x}-\mathbf{x}_{0}\right)\right]+\mathcal{O}\left(\dot{a}\left(t_{0}\right)\right),
\end{aligned}
$$

in a neighbourhood of the physical initial condition $x_{0}=$ $\left(t_{0}, \mathbf{x}_{0}\right)$, we understand that we may reach the correct flat limit when $a \rightarrow 1, \dot{a} \rightarrow 0, \cdots$ only if the initial time $t_{0}$ is chosen such that $a\left(t_{0}\right)=1$. This condition does not restrict the generality, as the scale factor is defined up to the scaling (15) for which we can at any time take $\sigma=a\left(t_{0}\right)$.

To avoid some difficulties related to the presence of the phase (89), it is convenient to redefine

$$
\begin{aligned}
f_{\mathbf{k}}\left(x_{c}\right) & =e^{-i \delta(\mathbf{k})} \hat{f}_{\mathbf{k}}\left(x_{c}-x_{c 0}\right) \\
& =\frac{1}{(2 \pi)^{3 / 2}} \frac{1}{\sqrt{2 k}} e^{-i k t_{c}+i \mathbf{k} \cdot \mathbf{x}_{c}}, \\
a(\mathbf{k}, \lambda) & =e^{i \delta(\mathbf{k})} \hat{a}(\mathbf{k}, \lambda),
\end{aligned}
$$

by substituting $\hat{f}_{\mathbf{k}}\left(x_{c}\right) \hat{a}(\mathbf{k}, \lambda)=f_{\mathbf{k}}\left(x_{c}\right) a(\mathbf{k}, \lambda)$ in Eq. (87) and denoting simply $A^{c}\left(x_{c}-x_{c 0}\right)=A^{c}\left(x_{c}\right)$.

The functions $f_{\mathbf{k}}(x)$ are assumed to be of positive frequencies, while those of negative frequencies are $f_{\mathbf{k}}(x)^{*}$. These solutions satisfy the orthonormalization relations [10]

$$
\left.\left(f_{\mathbf{k}}, f_{\mathbf{k}^{\prime}}\right)=-\left(f_{\mathbf{k}}^{*}, f_{\mathbf{k}^{\prime}}^{*}\right)=\delta^{3}\left(\mathbf{k}-\mathbf{k}^{\prime}\right),\right)
$$




$$
\left(f_{\mathbf{k}}, f_{\mathbf{k}^{\prime}}^{*}\right)=0
$$

and the completeness condition

$i \int \mathrm{d}^{3} k f_{\mathbf{k}}^{*}\left(t_{c}, \mathbf{x}_{c}\right) \stackrel{\leftrightarrow}{\partial_{t_{c}}} f_{\mathbf{k}}\left(t_{c}, \mathbf{x}^{\prime}\right)=\delta^{3}\left(\mathbf{x}_{c}-\mathbf{x}_{c}^{\prime}\right)$

with respect to the Hermitian form (74).

The polarization vectors $\mathbf{e}\left(\mathbf{n}_{k}, \lambda\right)$ in the Coulomb gauge must be orthogonal to the momentum direction,

$\mathbf{k} \cdot \mathbf{e}\left(\mathbf{n}_{k}, \lambda\right)=0$

for any polarization $\lambda= \pm 1$. We remind the reader that the polarization can be defined in different manners independent of the form of the scalar solutions $f_{\mathbf{k}}$. In general, the polarization vectors have c-number components which must satisfy [17]

$$
\begin{aligned}
\mathbf{e}\left(\mathbf{n}_{k}, \lambda\right) \cdot \mathbf{e}\left(\mathbf{n}_{k}, \lambda^{\prime}\right)^{*} & =\delta_{\lambda \lambda^{\prime}}, \\
\sum_{\lambda} e_{i}\left(\mathbf{n}_{k}, \lambda\right) e_{j}\left(\mathbf{n}_{k}, \lambda\right)^{*} & =\delta_{i j}-\frac{k^{i} k^{j}}{k^{2}} .
\end{aligned}
$$

In general, we consider the circular polarization for which the supplementary conditions $\mathbf{e}\left(\mathbf{n}_{k}, \lambda\right)^{*} \wedge \mathbf{e}\left(\mathbf{n}_{k}, \lambda\right)=i \lambda \mathbf{n}_{k}$ and $i \mathbf{n}_{k} \wedge \mathbf{e}\left(\mathbf{n}_{k}, \lambda\right)=\lambda \mathbf{e}\left(\mathbf{n}_{k}, \lambda\right)$ are fulfilled.

We thus obtained the mode expansion of the electromagnetic potential in terms of transverse plane waves of given momentum and helicity that form the momentum-helicity basis. The functions

$w_{i(\mathbf{k}, \lambda)}=e_{i}\left(\mathbf{n}_{k}, \lambda\right) f_{\mathbf{k}}$

are of positive frequencies, while those of negative frequencies are their complex conjugated, $w_{i(\mathbf{k}, \lambda)}^{*}$.

\subsection{Quantization and propagators}

The Coulomb gauge in the conformal frame considered above is just the framework we need for performing the quantization in a canonical manner as in special relativity [10]. We assume that the potential (87) and the wave functions $a$ become field operators, $A^{c} \rightarrow \mathcal{A}^{c}=\left(\mathcal{A}^{c}\right)^{\dagger}, a \rightarrow \mathfrak{a}$ and $a^{*} \rightarrow \mathfrak{a}^{\dagger}$. As in the flat case, we postulate the commutation relations in the momentum representation from which the non-vanishing ones are [10]

$\left[\mathfrak{a}(\mathbf{k}, \lambda), \mathfrak{a}^{\dagger}\left(\mathbf{k}^{\prime}, \lambda^{\prime}\right)\right]=\delta_{\lambda \lambda^{\prime}} \delta^{3}\left(\mathbf{k}-\mathbf{k}^{\prime}\right)$.

Then we verify that the field $\mathcal{A}^{c}$ is quantized according to the canonical rule

$$
\begin{aligned}
{\left[\mathcal{A}_{i}^{c}\left(t_{c}, \mathbf{x}_{c}\right), \pi_{c}^{j}\left(t_{c}, \mathbf{x}_{c}^{\prime}\right)\right] } & =\left[\mathcal{A}_{i}^{c}\left(t_{c}, \mathbf{x}_{c}\right), \partial_{t_{c}} \mathcal{A}_{j}^{c}\left(t_{c}, \mathbf{x}_{c}^{\prime}\right)\right] \\
& =i \delta_{i j}^{t r}\left(\mathbf{x}_{c}-\mathbf{x}_{c}^{\prime}\right),
\end{aligned}
$$

where the operator

$\pi_{c}^{j}=\sqrt{g} \frac{\delta \mathcal{L}\left(\mathcal{A}^{c}\right)}{\delta\left(\partial_{t_{c}} \mathcal{A}_{j}^{c}\right)}=\partial_{t_{c}} \mathcal{A}_{j}^{c}$ is the momentum density in the Coulomb gauge, while

$\delta_{i j}^{t r}\left(\mathbf{x}_{c}\right)=\frac{1}{(2 \pi)^{3}} \int \mathrm{d}^{3} q\left(\delta_{i j}-\frac{q^{i} q^{j}}{q^{2}}\right) e^{i \mathbf{q} \cdot \mathbf{x}_{c}}$

is the well-known transverse $\delta$-function [17] arising from Eq. (98). Under such circumstances, we may assume that there exists a unique vacuum state $|0\rangle$ of the Fock space such that

$\mathfrak{a}(\mathbf{k}, \lambda)|0\rangle=0, \quad\langle 0| \mathfrak{a}^{\dagger}(\mathbf{k}, \lambda)=0$.

The sectors with a given number of particles have to be constructed using the standard methods, thereby obtaining the generalized momentum-helicity basis.

In conformal frames, we may look for the Green functions related to the partial commutator functions (of positive or negative frequencies) defined as

$D_{i j}^{c( \pm)}\left(x_{c}-x_{c}^{\prime}\right)=-i\left[\mathcal{A}_{i}^{c( \pm)}(x), \mathcal{A}_{j}^{c( \pm) \dagger}\left(x^{\prime}\right)\right]$

while the total one reads $D_{i j}^{c}=D_{i j}^{c(+)}+D_{i j}^{c(-)}$. These functions are solutions of the field equation in both sets of variables and obey $\left[D_{i j}^{c( \pm)}\right]^{*}=D_{i j}^{c(\mp)}$ such that $D_{i j}^{c}$ results in a real function. Thus it is enough to focus only on the functions of positive frequencies [10],

$$
\begin{gathered}
i D_{i j}^{c(+)}\left(x_{c}-x_{c}^{\prime}\right)=\int \mathrm{d}^{3} k f_{\mathbf{k}}\left(x_{c}\right) f_{\mathbf{k}}\left(x_{c}^{\prime}\right)^{*}\left(\delta_{i j}-\frac{k^{i} k^{j}}{k^{2}}\right) \\
=\frac{1}{(2 \pi)^{3}} \int \frac{\mathrm{d}^{3} k}{2 k}\left(\delta_{i j}-\frac{k^{i} k^{j}}{k^{2}}\right) e^{i \mathbf{k} \cdot\left(\mathbf{x}_{c}-\mathbf{x}_{c}^{\prime}\right)-i k\left(t_{c}-t_{c}^{\prime}\right)},
\end{gathered}
$$

which have the same form and properties as in the flat case. For example, at equal time we have

$$
\left.\partial_{t_{c}} D_{i j}^{c(+)}\left(t_{c}-t_{c}^{\prime}, \mathbf{x}_{c}-\mathbf{x}_{c}^{\prime}\right)\right|_{t_{c}^{\prime}=t_{c}}=-\frac{1}{2} \delta_{i j}^{t r}\left(\mathbf{x}_{c}-\mathbf{x}_{c}^{\prime}\right) .
$$

Using the above functions defined in conformal frames, we can construct different transverse Green functions, $G_{i j}^{c}(x)=G_{j i}^{c}(x)$, which are solutions of the Green equation,

$$
\left(\partial_{t_{c}}^{2}-\Delta_{c}\right) G_{i j}^{c}\left(x_{c}-x_{c}^{\prime}\right)=-\delta\left(t_{c}-t_{c}^{\prime}\right) \delta_{i j}^{t r}\left(\mathbf{x}_{c}-\mathbf{x}_{c}^{\prime}\right),
$$

and satisfy $\partial_{x_{c}^{i}} G_{. j}^{c i} \cdot\left(x_{c}\right)=0$. Of special interest are the retarded $(\mathrm{R})$, advanced $(\mathrm{A})$ and Feynman $(\mathrm{F})$ propagators defined as [10]

$$
\begin{aligned}
& D_{i j}^{c R}\left(x_{c}-x_{c}^{\prime}\right)=\theta\left(t_{c}-t_{c}^{\prime}\right) D_{i j}^{c}\left(x_{c}-x_{c}^{\prime}\right), \\
& D_{i j}^{c A}\left(x_{c}-x_{c}^{\prime}\right)=-\theta\left(t_{c}^{\prime}-t_{c}\right) D_{i j}^{c}\left(x_{c}-x_{c}^{\prime}\right), \\
& D_{i j}^{c F}\left(x_{c}-x_{c}^{\prime}\right)=-i\left\langle 0\left|T\left[\mathcal{A}_{i}^{c}\left(x_{c}\right) \mathcal{A}_{j}^{c}\left(x_{c}^{\prime}\right)\right]\right| 0\right\rangle \\
& \quad=\theta\left(t_{c}-t_{c}^{\prime}\right) D_{i j}^{c(+)}\left(x_{c}-x_{c}^{\prime}\right)-\theta\left(t_{c}^{\prime}-t_{c}\right) D_{i j}^{c(-)}\left(x_{c}-x_{c}^{\prime}\right) .
\end{aligned}
$$

It is not difficult to verify that all these functions satisfy Eq. (108) if we use the identity $\partial_{t}^{2}[\theta(t) f(t)]=\delta(t) \partial_{t} f(t)$ and 
Eq. (107). We conclude that the quantization procedure in conformal frames is similar to that applied in special relativity. The resulting Green functions depend only on four variables, for which we may use the Fourier integral representation in the complex $k^{0}$-plane as in the usual QED in the Minkowski space-time.

However, these properties do not hold in the physical frames where the commutator and Green functions are no longer functions of the differences between the coordinates of the two points, depending on these coordinates separately. For example, according to Eqs. (75) and (76), the commutator functions

$D_{i j}^{( \pm)}\left(x, x^{\prime}\right)=-i\left[\mathcal{A}_{i}^{( \pm)}(x), \mathcal{A}_{j}^{( \pm) \dagger}\left(x^{\prime}\right)\right]$

take the form

$D_{00}\left(x, x^{\prime}\right)=\frac{\dot{a}(t) \dot{a}\left(t^{\prime}\right)}{a(t)^{2} a\left(t^{\prime}\right)^{2}} x^{i} x^{j} D_{i j}^{c}\left(x, x^{\prime}\right)$,

$D_{0 j}\left(x, x^{\prime}\right)=-\frac{\dot{a}(t)}{a(t)^{2} a\left(t^{\prime}\right)} x^{i} D_{i j}^{c}\left(x, x^{\prime}\right)$,

$D_{j 0}\left(x, x^{\prime}\right)=-\frac{\dot{a}\left(t^{\prime}\right)}{a(t) a\left(t^{\prime}\right)^{2}} x^{i} D_{j i}^{c}\left(x, x^{\prime}\right)$,

$D_{i j}\left(x, x^{\prime}\right)=\frac{1}{a(t)^{2} a\left(t^{\prime}\right)^{2}} D_{i j}^{c}\left(x, x^{\prime}\right)$,

which hold for any function $D_{i j}^{c( \pm)}$ or $D_{i j}^{c}$ defined above, in which we substitute the physical coordinates as

$D_{i j}^{c}\left(x, x^{\prime}\right)=D_{i j}^{c}\left(t_{c}(t)-t_{c}\left(t^{\prime}\right), \frac{x}{a(t)}-\frac{x^{\prime}}{a\left(t^{\prime}\right)}\right)$.

These functions give rise to Green functions which do not have Fourier integral representations. Therefore, for calculating Feynman diagrams, we have to use the simpler formalism in conformal frames, resorting to the physical ones only for interpreting the final results.

\subsection{One-particle operators}

After quantization, we can define the one-particle operators corresponding to the Killing vectors generating the conserved quantities (55). The constants $C_{a}$ become the one-particle operators,

$\mathcal{X}_{a}=\frac{1}{2} \delta_{i j}:\left(\mathcal{A}_{i}^{c},\left(X_{a} \mathcal{A}_{j}^{c}\right)\right): \forall X_{a} \in e(3)$,

calculated respecting the normal ordering of the operator products $[16,17]$. These operators have the usual algebraic properties

$$
\begin{aligned}
{\left[\mathcal{X}_{a}, \mathcal{A}_{i}^{c}\left(x_{c}\right)\right] } & =-\left(X_{a} \mathcal{A}^{c}\right)_{i}\left(x_{c}\right), \\
{\left[\mathcal{X}_{a}, \mathcal{X}_{a^{\prime}}\right] } & =\frac{1}{2} \delta_{i j}:\left(\mathcal{A}_{i}^{c},\left(\left[X_{a}, X_{a^{\prime}}\right] \mathcal{A}^{c}\right)_{j}\right),
\end{aligned}
$$

resulting from their definitions under the canonical quantization adopted here.
The principal conserved one-particle operators are the components of momentum operator,

$\mathcal{P}^{l}=\frac{1}{2} \delta_{i j}:\left(\mathcal{A}_{i}^{c},\left(\hat{P}^{l} \mathcal{A}^{c}\right)_{j}\right):=\int \mathrm{d}^{3} k k^{l} \sum_{\lambda} \mathfrak{a}^{\dagger}(\mathbf{k}, \lambda) \mathfrak{a}(\mathbf{k}, \lambda)$,

and the Pauli-Lubanski operator,

$\mathcal{W}=\frac{1}{2} \delta_{i j}:\left(\mathcal{A}_{i}^{c},\left(\hat{W} \mathcal{A}^{c}\right)_{j}\right):=\int \mathrm{d}^{3} k k \sum_{\lambda} \lambda \mathfrak{a}^{\dagger}(\mathbf{k}, \lambda) \mathfrak{a}(\mathbf{k}, \lambda)$,

which form the complete system of commuting operators $\left\{\mathcal{P}^{i}, \mathcal{W}\right\}$ determining the momentum-helicity basis of the Fock space. The components of the total angular momentum, $\mathcal{J}_{i}$, have more complicated mode expansions that we do not present here, since these are not involved in the applications we discuss below.

Other conserved one-particle operators can be defined giving their mode expansion directly as in the simplest case of the operator of number of particles,

$\mathcal{N}=\int \mathrm{d}^{3} k \sum_{\lambda} \mathfrak{a}^{\dagger}(\mathbf{k}, \lambda) \mathfrak{a}(\mathbf{k}, \lambda)$,

or the conserved scalar momentum

$\mathcal{P}=\int \mathrm{d}^{3} k k \sum_{\lambda} \mathfrak{a}^{\dagger}(\mathbf{k}, \lambda) \mathfrak{a}(\mathbf{k}, \lambda)$,

which commute with $\mathcal{P}^{i}, \mathcal{J}_{i}$ and $\mathcal{W}$.

The conserved one-particle operators derived above are independent on the frame we use such that we have the opportunity to derive them in the conformal frame where the calculations are simpler. However, other observables that may depend on time and space coordinates must be considered only in the associated physical frame. For example, Eqs. (75) and (76) help us to derive the field operators $\mathcal{A}_{\mu}$ giving the field strength operators

$\mathcal{F}^{\alpha \beta}=\mathcal{F}^{\alpha \beta^{\dagger}}=g^{\alpha \mu} g^{\beta v}\left(\partial_{\mu} \mathcal{A}_{v}-\partial_{\nu} \mathcal{A}_{\mu}\right)$,

and of the conserved stress-energy operator

$\mathcal{T}^{\mu \nu}=: \mathcal{F}_{\cdot \alpha}^{\mu \cdot} \mathcal{F}^{\alpha \nu}+\frac{1}{4} g^{\mu \nu} \mathcal{F}^{\alpha \beta} \mathcal{F}_{\alpha \beta}$,

which must be calculated respecting the normal ordering of operator products [16]. All these operators are now physical observables for which we can derive the expectation values and dispersions in concrete applications.

We now have the field and one-particle operators we need for building our quantum theory, but some important operators are missing, such as for example the energy operator. This is because the energy is not conserved in the manifolds 
$(M, a)$ apart from the particular case of the de Sitter expanding universe [12]. Under such circumstances, the solution is to introduce an ad hoc energy operator based only on the correspondence principle applied to the energy (29) and peculiar momentum (32). We thus define the time-dependent energy, $\mathcal{H}(t)$, and peculiar momentum, $\mathcal{K}^{i}(t)$, operators as

$\mathcal{H}(t)=\frac{1}{a(t)} \mathcal{P}$

$\mathcal{K}^{i}(t)=\frac{1}{a(t)} \mathcal{P}^{i}$

where $\mathcal{P}$ and $\mathcal{P}^{i}$ are the conserved one-particle operators defined by Eqs. (124) and (121), respectively. A difficult task could be to relate this energy operator to the components of the stress-energy operator (126) and a suitable vector field $V$ such that

$\mathcal{H}(t)=-\int \mathrm{d}^{3} x \mathcal{T}(x)^{0 v} V_{v}(x)$.

Note that in the case of the de Sitter space-time, the operator $\mathcal{H}(t)$ is different from that of conserved energy, $\mathcal{H}_{d S}$, which does not commute with $\mathcal{P}$ and $\mathcal{P}^{i}$. These two energy operators coincide, $\mathcal{H}\left(t_{0}\right)=\mathcal{H}_{d S}$, only in the observer's origin at the time $t_{0}$ when $a\left(t_{0}\right)=1$ [12].

\section{Propagation}

We now have all the elements we need to study the propagation of the photons in the space-times $(M, a)$, where we revisit the problem of two observers, presented in Sec. (2.3), to analyse how a quantum beam can be prepared by the observer $O^{\prime}$ and then detected by $O$. As we proceeded in the de Sitter case [13], we start our study in the conformal frames of these observers, $\left\{t_{c}^{\prime}, \mathbf{x}_{c}^{\prime}\right\}_{O^{\prime}}$ and $\left\{t_{c}, \mathbf{x}_{c}\right\}_{O}$, respectively, where the calculations are simpler, but finally we analyse how the detected photon is measured by $O$ in its physical frame $\{t, \mathbf{x}\}_{O}$. We simplify the geometry by choosing the space axes of these frames parallel to the orthonormal frame $\left\{\mathbf{e}_{1}, \mathbf{e}_{2}, \mathbf{e}_{3}\right\}$ such that $\mathbf{n}=\mathbf{e}_{3}$ gives the direction $O^{\prime} O$.

\subsection{Prepared wave packet}

Following the method of Ref. [12], we consider the simple model of the one-particle wave packet produced by the oneparticle states,

$|\alpha\rangle=\int \mathrm{d}^{3} k \sum_{\lambda} \alpha_{\lambda}(\mathbf{k}) \mathfrak{a}^{\dagger}(\mathbf{k}, \lambda)|0\rangle$

defined by the square integrable functions in momentum representation $\alpha_{\lambda}(\mathbf{k})$ which must satisfy the normalization con- dition

$\langle\alpha \mid \alpha\rangle=\int \mathrm{d}^{3} k \sum_{\lambda}\left|\alpha_{\lambda}(\mathbf{k})\right|^{2}=1$.

Bearing in mind the dependence of the plane waves on the initial conditions, we chose functions of the form

$\alpha_{\lambda}(\mathbf{k})=e^{i \delta(\mathbf{k})} \hat{\alpha}_{\lambda}(\mathbf{k})$,

where $\delta(\mathbf{k})$ is the phase (89) while $\hat{\alpha}_{\lambda}=\hat{\alpha}_{\lambda}^{*}$ are real-valued functions. The corresponding 'wave functions' in Coulomb gauge

$A_{i}^{c}\left(x_{c}\right)=\left\langle 0\left|\mathcal{A}_{i}^{c}\left(x_{c}\right)\right| \alpha\right\rangle=\int \mathrm{d}^{3} k \sum_{\lambda} e_{i}\left(\mathbf{n}_{k}, \lambda\right) f_{\mathbf{k}}\left(x_{c}\right) \alpha_{\lambda}(\mathbf{k})$

define the normalized wave packets whose norm,

$\left\|A^{c}\right\|^{2}=\delta_{i j}\left(A_{i}^{c}, A_{j}^{c}\right)=\int \mathrm{d}^{3} k \sum_{\lambda}\left|\alpha_{\lambda}(\mathbf{k})\right|^{2}=1$,

results from Eqs. (93) and (94). Note that the components $A_{i}^{c}$ are now complex-valued functions which have to be manipulated carefully in order to lead to real-valued physical quantities.

Another advantage of this model is that the expectation values in the state $|\alpha\rangle$ of the operators having the form (118) can be calculated simply by using the identity

$\langle\alpha|\mathcal{X}| \alpha\rangle=\delta_{i j}\left(A_{i}^{c}, X A_{j}^{c}\right)$.

For the other operators which do not have this form, such as for example $\mathcal{N}$ and $\mathcal{P}$, we have to use the algebra of the field operators. Thus we obtain the following expectation values for the operators which are diagonal in the momentumhelicity basis,

$$
\begin{aligned}
\left\langle\alpha\left|\left(\mathcal{P}^{i}\right)^{n}\right| \alpha\right\rangle & =\delta_{i j}\left(A_{i}^{c},\left(\hat{P}^{i}\right)^{n} A_{j}^{c}\right) \\
& =\int \mathrm{d}^{3} k\left(k^{i}\right)^{n} \sum_{\lambda} \hat{\alpha}_{\lambda}(\mathbf{k})^{2}, \\
\left\langle\alpha\left|\mathcal{W}^{n}\right| \alpha\right\rangle & =\delta_{i j}\left(A_{i}^{c}, \hat{W}^{n} A_{j}^{c}\right) \\
& =\int \mathrm{d}^{3} k \sum_{\lambda} \lambda^{n} \hat{\alpha}_{\lambda}(\mathbf{k})^{2},
\end{aligned}
$$

while for the energy operator we directly calculate

$$
\left\langle\alpha\left|\mathcal{H}(t)^{n}\right| \alpha\right\rangle=\frac{1}{a(t)^{n}} \int \mathrm{d}^{3} k k^{n} \sum_{\lambda} \hat{\alpha}_{\lambda}(\mathbf{k})^{2} .
$$

All these expressions will help us to derive the expectation values (135) and the dispersions of the principal observables, $X$, according to the well-known rule [15]

$\operatorname{disp} X=\Delta X^{2}=\left\langle\alpha\left|\mathcal{X}^{2}\right| \alpha\right\rangle-\langle\alpha|\mathcal{X}| \alpha\rangle^{2}$, 
that will be used in evaluating the accuracy of the measurements.

We now assume that the observer $O^{\prime}$ prepares the state $|\alpha\rangle$ in its proper co-moving frame $\left\{t_{c}, \mathbf{x}_{c}^{\prime}\right\}_{O^{\prime}}$ at the initial time

$t_{0} \rightarrow t_{c 0}=t_{c}\left(t_{0}\right)$,

when $a\left(t_{0}\right)=1$, and consequently the conformal and physical space coordinates coincide. Then the principal parameters measured by $O^{\prime}$ are the expectation values of energy, $E^{\prime}$, momentum components, $P^{\prime i}$, and polarization, $W^{\prime}$. These quantities can be calculated by using the above identities, taking into account that now the packet is defined in $O^{\prime}$ by the functions (132) with the phase

$\mathbf{x}_{c 0}^{\prime}=0 \rightarrow \delta^{\prime}(\mathbf{k})=k t_{c 0}$.

Therefore, we may write the prepared wave packet as

$A_{i}^{c \prime}\left(x_{c}\right)=\frac{1}{(2 \pi)^{3 / 2}} \int \frac{\mathrm{d}^{3} k}{\sqrt{2 k}} e^{-i k\left(t_{c}-t_{c 0}\right)+i \mathbf{k} \cdot \mathbf{x}_{c}} \sum_{\lambda} e_{i}\left(\mathbf{n}_{k}, \lambda\right) \hat{\alpha}_{\lambda}(\mathbf{k})$.

The expectation value of the energy is $E^{\prime}=P^{\prime}$, since $a\left(t_{0}\right)=$ 1 , while the expectation values of the other observables have to be calculated by using Eqs. (136), (137) and (138) for $n=1$. The dispersions can be derived by using the same identities (with $n=2$ ) and the definition (139). We observe that in the conformal frame of $O^{\prime}$, all these expectation values and dispersions have the same forms as in Minkowski spacetime.

In applications, it is convenient to introduce the polarization angle $\sigma(\mathbf{k})$ substituting

$$
\begin{gathered}
\hat{\alpha}_{\lambda=1}(\mathbf{k})=\hat{\alpha}(\mathbf{k}) \cos \sigma\left(\mathbf{n}_{k}\right), \\
\hat{\alpha}_{\lambda=-1}(\mathbf{k})=\hat{\alpha}(\mathbf{k}) \sin \sigma\left(\mathbf{n}_{k}\right),
\end{gathered}
$$

since then we can use identities as

$$
\begin{aligned}
\sum_{\lambda} \hat{\alpha}_{\lambda}(\mathbf{k})^{2} & =\hat{\alpha}(\mathbf{k})^{2}, \\
\sum_{\lambda} \lambda \hat{\alpha}_{\lambda}(\mathbf{k})^{2} & =\hat{\alpha}(\mathbf{k})^{2} \cos 2 \sigma\left(\mathbf{n}_{k}\right),
\end{aligned}
$$$$
\ldots
$$

taking into account that the new real-valued function $\hat{\alpha}(\mathbf{k})$ is normalized,

$$
\int \mathrm{d}^{3} k \hat{\alpha}(\mathbf{k})^{2}=1
$$

Thus we can say that any wave packet is determined by two scalar functions $\hat{\alpha}(\mathbf{k})$ and $\sigma\left(\mathbf{n}_{k}\right)$.

In general, when $\hat{\alpha}(\mathbf{k})$ is an arbitrary function of $\mathbf{k}$, we say that the packet is anisotropic. In the special case when this function has spherical symmetry, $\hat{\alpha}(\mathbf{k})=\hat{\alpha}(k)$, we have an isotropic packet for which it is convenient to use the spherical coordinates in momentum space, $(k, \theta, \phi)$, corresponding to the Cartesian ones, $\left(k^{1}, k^{2}, k^{3}\right)$. The principal property of the isotropic packets is that the expectation values of the momentum components vanish such that the only relevant quantity remains the radial momentum, whose expectation value reads

$P^{\prime}=E^{\prime}=4 \pi \int_{0}^{\infty} \mathrm{d} k k^{3} \hat{\alpha}(k)^{2}$.

Note that $P^{\prime}$ may not be confused with the norm of the vector formed by the expectation values of the momentum components, $\left(P^{\prime 1}, P^{\prime 2}, P^{\prime 3}\right)$, as in the isotropic case these vanish. In this approach, the polarization remains arbitrary, $\sigma\left(\mathbf{n}_{k}\right)=\sigma(\theta, \phi)$, such that

$W^{\prime}=\frac{1}{4 \pi} \int_{S^{2}} \mathrm{~d} \Omega \cos 2 \sigma(\theta, \phi)$,

where $\mathrm{d} \Omega=d(\cos \theta) \mathrm{d} \phi$ is the measure on the sphere $S^{2}$.

\subsection{Detected wave packet}

Once the wave packet is prepared, this evolves causally until an ideal apparatus measures some of its parameters. In what follows we consider a similar experiment as in Ref. [12], assuming that the apparatus selects only the momenta included in a desired domain $\Delta \subset \mathbb{R}_{k}^{3}$. For this setting, we must use a suitable projection operator $\Lambda_{\Delta}=\Lambda_{\Delta}^{\dagger}$ (satisfying $\Lambda_{\Delta}^{2}=\Lambda_{\Delta}$ ) that can be represented as

$\Lambda_{\Delta}=|0\rangle\left\langle 0\left|+\int_{\Delta} \mathrm{d}^{3} k \sum_{\lambda} \mathfrak{a}^{\dagger}(\mathbf{k}, \lambda)\right| 0\right\rangle\langle 0| \mathfrak{a}(\mathbf{k}, \lambda)+\cdots$,

where the integral is restricted to the domain $\Delta$. During the experiment, this operator filters only the momenta $\mathbf{k} \in \Delta$, transforming the state of the system as $|\alpha\rangle \rightarrow \Lambda_{\Delta}|\alpha\rangle$ and giving the new wave packet

$\left\langle 0\left|\Lambda_{\Delta} \mathcal{A}_{i}^{c}\left(x_{c}\right)\right| \alpha\right\rangle=\int_{\Delta} \mathrm{d}^{3} k \sum_{\lambda} e_{i}\left(\mathbf{n}_{k}, \lambda\right) f_{\mathbf{k}}\left(x_{c}\right) \alpha_{\lambda}(\mathbf{k})$.

In general, the new state $\Lambda_{\Delta}|\alpha\rangle$ is no longer normalized, such that we must redefine the expectation value $\langle\mathcal{X}\rangle$ of a one-particle operator $\mathcal{X}$ as [15]

$\langle\mathcal{X}\rangle=\frac{\left\langle\alpha\left|\Lambda_{\Delta} \mathcal{X}\right| \alpha\right\rangle}{\left\langle\alpha\left|\Lambda_{\Delta}\right| \alpha\right\rangle}$,

taking into account that our one-particle operators commute with $\Lambda_{\Delta}$. The quantity

$\left\langle\alpha\left|\Lambda_{\Delta}\right| \alpha\right\rangle=\int_{\Delta} \mathrm{d}^{3} k \sum_{\lambda}\left|\alpha_{\lambda}(\mathbf{k})\right|^{2} \leq 1$,

gives the probability $P_{\Delta}=\left|\left\langle\alpha\left|\Lambda_{\Delta}\right| \alpha\right\rangle\right|^{2}$ of measuring any momentum $\mathbf{k} \in \Delta$. Obviously, when we can measure the whole continuous spectrum, $\Delta=\mathbb{R}_{k}^{3}$, then $\Lambda_{\Delta} \rightarrow \mathbf{1}, P_{\Delta}=1$ and $\langle\mathcal{X}\rangle=\langle\alpha|\mathcal{X}| \alpha\rangle$. 
The detector of $O$ must select only the photons coming from the source $O^{\prime}$ whose momenta are parallel to $\mathbf{e}_{3}$. This means that the domain of momenta measured by $O$ is [12]

$\Delta=\left\{\mathbf{k} \mid-\frac{\Delta k}{2} \leq k^{1} \leq \frac{\Delta k}{2},-\frac{\Delta k}{2} \leq k^{2} \leq \frac{\Delta k}{2}, k^{3}>0\right\}$

where $\Delta k$ is a small quantity. Then we may evaluate the integrals over $\Delta$ as

$$
\begin{aligned}
\int_{\Delta} \mathrm{d}^{3} k F(\mathbf{k}) & =\int_{-\frac{\Delta k}{2}}^{\frac{\Delta k}{2}} \mathrm{~d} k^{1} \int_{-\frac{\Delta k}{2}}^{\frac{\Delta k}{2}} \mathrm{~d} k^{2} \int_{-\infty}^{0} \mathrm{~d} k^{3} F\left(k^{1}, k^{2}, k^{3}\right) \\
& \simeq(\Delta k)^{2} \int_{0}^{\infty} \mathrm{d} k F(0,0, k)
\end{aligned}
$$

according to the mean value theorem. Thus we can calculate the quantity

$\left\langle\alpha\left|\Lambda_{\Delta}\right| \alpha\right\rangle=\int_{\Delta} \mathrm{d}^{3} k \sum_{\lambda} \hat{\alpha}_{\lambda}(\mathbf{k})^{2}=(\Delta k)^{2} \kappa$,

where

$\kappa=\int_{0}^{\infty} \mathrm{d} k \sum_{\lambda} \hat{\alpha}_{\lambda}(0,0, k)^{2}$.

Furthermore, we observe that the new wave packet (150) behaves as an one-dimensional packet that can be redefined as [12]

$A_{i}^{c}\left(x_{c}\right)=\frac{1}{\sqrt{\kappa}} \int_{0}^{\infty} \mathrm{d} k \sum_{\lambda} e_{i}(\lambda) \tilde{f}_{\mathbf{k}}\left(x_{c}^{3}\right) e^{i \delta(\mathbf{k})} \hat{\alpha}_{\lambda}(0,0, k)$

where the new functions

$\tilde{f}_{\mathbf{k}}\left(x_{c}^{3}\right)=\frac{1}{\sqrt{2 \pi}} \frac{1}{\sqrt{2 k}} e^{-i k t_{c}+i k x_{c}^{3}}$,

are orthonormal with respect to the new Hermitian form

$$
(\tilde{f} ; \tilde{g})=i \int \mathrm{d} x_{c}^{3} \tilde{f}^{*}\left(t_{c}, x_{c}^{3}\right) \stackrel{\leftrightarrow}{\partial_{t_{c}}} \tilde{g}\left(t_{c}, x_{c}^{3}\right)
$$

In our experiment, we select only the momenta oriented along $\mathbf{e}_{3}$ such that the polarizations vectors $\mathbf{e}( \pm 1)=\frac{1}{\sqrt{2}}\left(\mathbf{e}_{1} \pm i \mathbf{e}_{2}\right)$ are in the plane $\left\{\mathbf{e}_{1}, \mathbf{e}_{2}\right\}$. As the functions here $\hat{\alpha}_{\lambda}$ are those of Eq. (142), we may use the substitutions (143) and (144) that now read

$$
\hat{\alpha}_{\lambda=1}(0,0, k)=\hat{\alpha}(0,0, k) \cos \sigma,
$$

$\hat{\alpha}_{\lambda=-1}(0,0, k)=\hat{\alpha}(0,0, k) \sin \sigma$,

where the function $\hat{\alpha}$ satisfies

$$
\int_{0}^{\infty} \mathrm{d} k \hat{\alpha}(0,0, k)^{2}=\kappa
$$

as results from Eq. (156). Now, $\sigma=\sigma\left(\mathbf{e}_{3}\right)$ denotes the constant polarization angle of the direction $\mathbf{e}_{3}$ giving the polarization unit vector

$\mathbf{e}(\sigma)=\mathbf{e}(1) \cos \sigma+\mathbf{e}(-1) \sin \sigma$.

On the other hand, for the states measured in $O$, we must consider the translated phase

$\delta(\mathbf{k})=k t_{c 0}+\mathbf{k} \cdot \mathbf{d}$,

arriving at the final form of the potential of the onedimensional packet

$A_{i}^{c}\left(t_{c}, x_{c}^{3}\right)=e_{i}(\sigma) A(X), \quad X=t_{c}-t_{c 0}-x_{c}^{3}-d$,

having the fixed direction along the unit vector $\mathbf{e}(\sigma)$ and depending on the amplitude [12]

$A(X)=\frac{1}{\sqrt{2 \pi \kappa}} \int_{0}^{\infty} \frac{d k}{\sqrt{2 k}} e^{-i k X} \hat{\alpha}(0,0, k) \in \mathbb{C}$,

which encapsulates the principal integral we have to solve to study the propagation.

\subsection{Measurements in physical frames}

First of all, the observer $O$ records the expectation values and dispersions of the principal conserved observables which are the components of momentum, angular momentum and the Pauli-Lubanski operator. For both the observers situated on the null geodesic, the angular momentum vanishes such that $O$ remains with the expectation values and dispersions of the momentum components,

$$
\begin{aligned}
P^{3}=P & =\frac{1}{\kappa} \int_{0}^{\infty} \mathrm{d} k k \hat{\alpha}(0,0, k)^{2}, \quad P^{1}=P^{2}=0, \\
\operatorname{disp} P & =\frac{1}{\kappa} \int_{0}^{\infty} \mathrm{d} k k^{2} \hat{\alpha}(0,0, k)^{2}-P^{2},
\end{aligned}
$$

and the similar quantities of the Pauli-Lubanski operator,

$W=\cos 2 \sigma, \quad \operatorname{disp} W=\sin ^{2} 2 \sigma$.

For the energy operators we have to use the identity (138) that depends explicitly on time. Thus the expectation value of the emitted photon is $E_{i}=P$ since $a\left(t_{0}\right)=1$. To find the final energy of the detected photon, $E_{f}=a\left(t_{f}\right)^{-1} P$, we need to know the time $t_{f}$ when the photon is detected in $O$. We showed in Sec. (2.3) that $t_{f}$ can be derived by solving Eq. (41). Moreover, this is related to the redshift,

$a\left(t_{f}\right)=1+z$,

resulted from $\mathrm{Eq}(43)$ for $a\left(t_{0}\right)=1$.

Apart from the conserved quantities, $O$ can measure the parameters of the electromagnetic field in its proper physical frame, $\{t, \mathbf{x}\}_{O}$. As the potentials cannot be measured directly, we must look for relevant quantities such as the wave intensity or the density of energy which must now be derived in 
this frame. For simplicity, we consider the plane polarization fixing the polarization angle [13]

$\sigma\left(\mathbf{e}_{3}\right)=\frac{\pi}{4} \rightarrow \mathbf{e}(\sigma)=\mathbf{e}_{1}$,

so that we remain only with the component $A_{1}^{c}$ of the potential (165). In the physical frame, this generates the components (75) and (76) that now read

$$
\begin{aligned}
& A_{0}(t, \mathbf{x})=\frac{\partial x_{c}^{i}}{\partial t} A_{i}^{c}\left(t_{c}, x_{c}^{3}\right)=-\frac{\dot{a}(t)}{a(t)^{2}} x^{1} A(X), \\
& A_{1}(t, \mathbf{x})=\frac{\partial x_{c}^{i}}{\partial x^{1}} A_{i}^{c}\left(t_{c}, x_{c}^{3}\right)=\frac{1}{a(t)} A(X), \\
& A_{2}(t, \mathbf{x})=A_{3}(t, \mathbf{x})=0,
\end{aligned}
$$

where $A$ is the amplitude (166) depending on the new variable $X=t_{c}(t)-t_{c}\left(t_{0}\right)-\frac{x^{3}}{a(t)}-d$,

obtained from Eq. (165) after the substitution (9). These potentials satisfy the Maxwell equation and the Lorenz condition in the frame $\{t, \mathbf{x}\}_{O}$ where the field strength, $F^{\mu \nu}$, has the components

$F=\frac{1}{a(t)^{2}} \partial_{X} A(X)\left(\begin{array}{cccc}0 & -1 & 0 & 0 \\ 1 & 0 & \frac{\dot{a}(t)}{a(t)} x^{2} & p \\ 0 & -\frac{\dot{a}(t)}{a(t)} x^{2} & 0 & 0 \\ 0 & -p & 0 & 0\end{array}\right)$,

written with the notation $p=1+\frac{\dot{a}(t)}{a(t)} x^{3}$. The stress-energy tensor must be redefined as

$T^{\mu \nu}=F_{\cdot \alpha}^{\mu *} F^{\alpha \nu}-\frac{1}{4} g^{\mu v} F_{\cdot \alpha}^{\beta *} F_{\cdot \beta}^{\alpha}$,

as now we work with the complex-valued amplitude $A(X)$. We find that this has the components

$$
\begin{aligned}
T= & \frac{1}{a(t)^{4}}\left|\partial_{X} A(X)\right|^{2} \\
& \times\left(\begin{array}{cccc}
1 & \frac{\dot{a}(t)}{a(t)} x^{1} & \frac{\dot{a}(t)}{a(t)} x^{2} & p \\
\frac{\dot{a}(t)}{a(t)} x^{1} & \frac{\left.\dot{a}()^{2}\right)^{2}}{a(t)^{2}}\left(x^{1}\right)^{2} & \frac{\dot{a}(t)^{2}}{a(t)^{2}} x^{1} x^{2} & \frac{\dot{a}(t)}{a(t)} x^{1} p \\
\frac{\dot{a}(t)}{a(t)} x^{2} & \frac{\dot{a}(t)^{2}}{a(t)} x^{1} x^{2} & \frac{\dot{a}(t)^{2}}{a(t)^{2}}\left(x^{2}\right)^{2} & \frac{\dot{a}(t)}{a(t)} x^{2} p \\
p & \frac{\dot{a}(t)}{a(t)} x^{1} p & \frac{\dot{a}(t)}{a(t)} x^{2} p & p^{2}
\end{array}\right) .
\end{aligned}
$$

It is not difficult to verify that this satisfies the conservation rule $\nabla_{\mu} T^{\mu \nu}=0$.

To study the propagation of our wave packet, we follow the method of Ref. [13], focusing on the intensity which coincides with the density of energy,

$I(t, x)=\delta_{i j} F^{0 i^{*}} F^{0 j} \equiv T^{00}$,

introducing the factorization

$I(t, x)=\frac{1}{a(t)^{4}} I_{0}(X), \quad I_{0}(X)=\left|\partial_{X} A(X)\right|^{2}$, and re-denoting more simply, $x^{3} \rightarrow x$. Thus we separate the factor $a(t)^{-4}$ from the function $I_{0}(X)$ depending only on the amplitude (166). In Ref. [13] we showed that if we construct this amplitude by using only positive definite test functions $\alpha(\mathbf{k})$, then we have $\left|\partial_{X} A(X)\right| \leq\left|\partial_{X} A(X)\right|_{X=0}$. Consequently, the function $I_{0}(t, x)$ has an absolute (or global) maximum $I_{m}(t)$ in the point

$x_{m}(t)=a(t)\left[t_{c}(t)-t_{c}\left(t_{0}\right)-d\right]$,

where $X=0$. Remarkably, this is just the null geodesic (37) passing through $O^{\prime}$ and $O$. Once the wave packet is prepared at the time $t_{0}$ in the point $x_{m}\left(t_{0}\right)=-d$, its maximum propagates on this geodesic, with the velocity (44), arriving with the velocity $v\left(t_{f}\right)=1$ in $O$ (where $x_{m}\left(t_{f}\right)=0$ ) at the time $t_{f}$.

In expanding space-times $(M, a)$, the factor $a(t)^{-4}$ produces the decay of the maximum intensity from $I_{m}(0)=$ $I_{0}(0)$ up to

$I_{m}\left(t_{f}\right)=\frac{I_{0}(0)}{a\left(t_{f}\right)^{4}}=\frac{I_{0}(0)}{(1+z)^{4}}$,

as results from Eq. (170). Thus we may estimate the emitted maximum intensity, $I_{m}(0)$, in terms of the measured maximum intensity, $I_{m}\left(t_{f}\right)$, and redshift. Similarly, we deduce the distance $O^{\prime} O$ at the moment $t_{f}$,

$d\left(t_{f}\right)=d a\left(t_{f}\right)=(1+z) d$,

as predicted by Lemaitre's equation [29].

The space dispersion of the wave packet, $\delta x(t)$, measures the width of the function $I(t, X)$ at a given time. This depends on the width $\delta X$ of the function $I_{0}(X)$ that is a constant quantity depending on the profile of this function. Then, according to Eq. (173), we deduce that the physical dispersion,

$\delta x(t)=\delta X a(t)$,

increases from $\delta x\left(t_{0}\right)=\delta X$ up to $\delta x\left(t_{f}\right)=(1+z) \delta X$.

Thus we have completely described the propagation of the detected wave packets in the proper physical frame of $O$, pointing out their principal features, acceleration, decay and dispersion that cannot be observed in the Minkowski flat space-time. As was expected, these results are similar to those derived for the de Sitter expanding universe [13], which is a particular case of space-time $(M, a)$

\section{Horizon effect}

In Ref. [13] we studied the horizon effects as recorded by the observer $O$ in its proper physical frame where the event and apparent horizons of this geometry are static and coincide. We have seen that only the packets prepared inside this common horizon may be detected by the observer $O$, while those prepared on or beyond it never arrive in $O$, as was expected 
according to the general theory of the event horizons [30,31]. In other respects, we outlined in section (2.3) the effect produced by the dynamical apparent horizon which seems to be more interesting. Now we have the opportunity to analyse the horizon effects in concrete spatially flat FLRW spacetimes where the dynamical apparent and event horizons do not overlap each other as in the de Sitter case.

\subsection{Isotropic wave packet}

To test the horizon effects, we consider a simple isotropic wave packet for which it is convenient to use spherical coordinates in momentum space, $\mathbf{k}=(k, \theta, \phi)$. We assume that at the initial moment $t_{0}$ when $a\left(t_{0}\right)=1$, the observer $O^{\prime}$ prepares the wave packet in $x_{0}=-d$, defining the real-valued wave function in momentum representation (132) as

$\hat{\alpha}(k)=\frac{(2 \gamma)^{\gamma \bar{k}}}{2 \sqrt{\pi \Gamma(2 \gamma \bar{k})}} k^{\gamma \bar{k}-\frac{3}{2}} e^{-\gamma k}, \quad \gamma, \bar{k}>0$,

and keeping the plane polarization (171). This function is normalized,

$\int \mathrm{d}^{3} k \hat{\alpha}(k)^{2}=4 \pi \int_{0}^{\infty} \mathrm{d} k k^{2} \hat{\alpha}(k)^{2}=1$,

depending on the free parameter $\gamma$ and the expectation value of the radial momentum

$P^{\prime}=4 \pi \int_{0}^{\infty} \mathrm{d} k k^{3} \hat{\alpha}(k)^{2}=\bar{k}$,

whose dispersion can be derived simply as

$\operatorname{disp} P^{\prime}=4 \pi \int_{0}^{\infty} \mathrm{d} k k^{4} \hat{\alpha}(k)^{2}-\bar{k}^{2}=\frac{\bar{k}}{2 \gamma}$.

The observer $O$ measures the one-dimensional packet which must be re-normalized by using the constant

$\kappa=\int_{0}^{\infty} \mathrm{d} k \hat{\alpha}(k)^{2}=\frac{\gamma^{2}}{2 \pi(\gamma \bar{k}-1)(2 \gamma \bar{k}-1)}$.

This helps us to derive the expectation value of the momentum along the third axis

$P^{3}=P=\frac{1}{\kappa} \int_{0}^{\infty} \mathrm{d} k k \hat{\alpha}(k)^{2}=\bar{k}-\frac{1}{\gamma}=P^{\prime}-\frac{1}{\gamma}$,

and its dispersion

$$
\begin{aligned}
\operatorname{disp} P=\frac{1}{\kappa} \int_{0}^{\infty} \mathrm{d} k k^{2} \hat{\alpha}(k)^{2}-\bar{k}^{2} & =\frac{\bar{k}}{2 \gamma}-\frac{1}{2 \gamma^{2}} \\
& =\operatorname{disp} P^{\prime}-\frac{1}{2 \gamma^{2}},
\end{aligned}
$$

as observed by $O$.

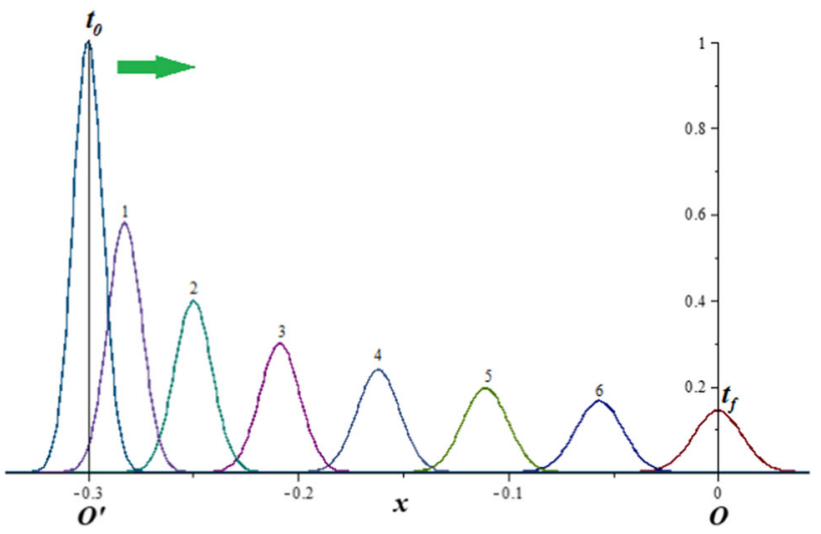

Fig. 1 Propagation of the wave packet with $\gamma=0.1$ and $\bar{k}=1000$ in the space-time with $s=0.3$ and $r_{a}\left(t_{0}\right)=\frac{1}{3}$. The packet is prepared in $O^{\prime}$ at the time $t_{0}=0.1$ and $d=0.3<r_{a}\left(t_{0}\right)$ and measured in $O$ at the time $t_{f}=0.5034$. We plot eight consecutive profiles of the maximum intensity $I(t, x)$ between $t_{0}$ and $t_{f}$ at equal time intervals, $\Delta t=0.0576$

The amplitude in the physical frame of the observer $O$ is given by Eq. (166) as

$$
\begin{aligned}
A_{L}(X)= & 2^{\gamma \bar{k}-2} \sqrt{\frac{(2 \gamma \bar{k}-2)(2 \gamma \bar{k}-1)}{\pi \Gamma(2 \gamma \bar{k})}} \\
& \times \Gamma(\gamma \bar{k}-1)\left(\frac{\gamma}{\gamma+i X}\right)^{\gamma \bar{k}-1} .
\end{aligned}
$$

With this amplitude we construct the intensity (178),

$I_{0}(X)=\left|\partial_{X} A_{L}(X)\right|^{2}=I_{0}\left(\frac{\gamma^{2}}{\gamma^{2}+X^{2}}\right)^{\gamma \bar{k}}$

where we denote

$I_{0}=I_{0}(0)=2^{2 \gamma \bar{k}-4} \frac{(2 \gamma \bar{k}-2)(2 \gamma \bar{k}-1)}{\pi \gamma^{2} \Gamma(2 \gamma \bar{k})} \Gamma(\gamma \bar{k})^{2}$.

The intensity (191) is the power of a genuine Cauchy-Lorentz distribution whose width,

$\delta X=2 \gamma \sqrt{2^{\gamma \bar{k}}-1}$,

determines the dispersion (182).

\subsection{Expanding space-times}

In what follows we consider, as examples, the expanding space-times $(M, a)$ with dynamical apparent horizons having scale factors of the form $a(t) \propto t^{s}$ with any $s>0$ [30]. It is known that these space-times are generated by the density and pressure

$\sigma=\frac{3}{8 \pi} \frac{s^{2}}{t^{2}}, \quad p=-\frac{1}{8 \pi} \frac{s(3 s-2)}{t^{2}}$,

that are singular in $t=0$ such that we must restrict the time domain to $t>0$. The important particular cases studied in 


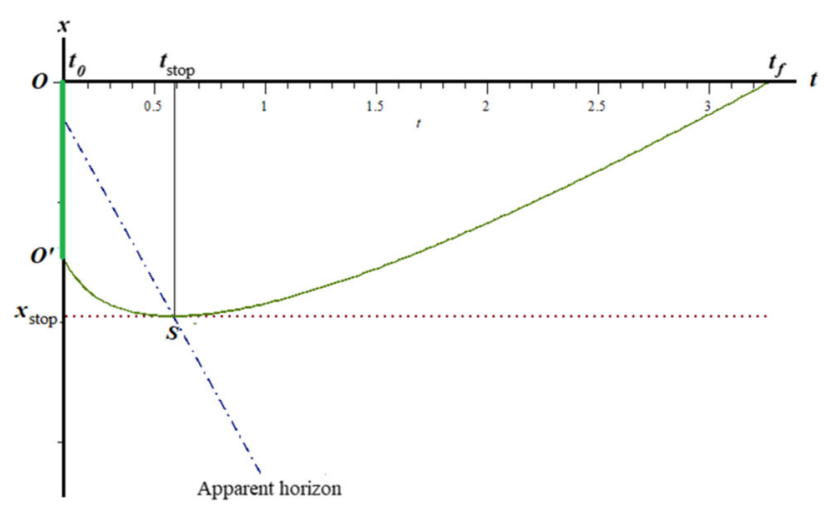

Fig. 2 Profile of the null geodesic $x_{m}(t)$ of the maximum intensity of the wave packet with $\gamma=0.1$ and $\bar{k}=1000$ in the space-time with $s=0.3$, and $r_{a}\left(t_{0}\right)=\frac{1}{3}$. The packet is prepared in $O^{\prime}$ at the time $t_{0}=0.1$ and $d=1>r_{e}\left(t_{0}\right)$ and measured in $O$ at the time $t_{f}=1.9504$. The maximum intensity stops in $x_{\text {stop }}=-1.164$ at the time $t_{\text {stop }}=0.3492$

cosmology are the radiation-dominant $\left(s=\frac{1}{2}\right)$ or matterdominant $\left(s=\frac{2}{3}\right.$ ) universes. Nevertheless, here we consider all the space-times with $s>0$ to point out the effect of the apparent horizons when an event horizon may or may not be present.

The scale factors of these space-times can be rescaled according to Eq. (15) as

$a(t)=\left(\frac{t}{t_{0}}\right)^{s}, \quad \frac{\dot{a}(t)}{a(t)}=\frac{s}{t}, \quad r_{a}(t)=\frac{t}{s}, \quad v_{a}=\frac{1}{s}$,

obtaining the Hubble function and the apparent horizon of the observer $O$ in its proper physical frame. This horizon is a sphere of radius $r_{a}(t)$ expanding with the constant velocity $v_{a}$. Looking then for event horizons, we observe that the integral (46) diverges for $s \leq 1$ such that there are no event horizons. However, for $s>1$, there exist event horizons at any time $t>0$ on the spheres of radius [30]

$r_{e}(t)=\int_{t}^{\infty} \mathrm{d} t^{\prime} \frac{t^{s}}{t^{\prime s}}=\frac{t}{s-1}, \quad v_{e}=\frac{1}{s-1}$,

resulted from from Eq. (46). Therefore, in this case the observer $O$ has at the time $t$ the apparent horizon (195) and, in addition, a dynamical event horizon at $r_{e}(t)>r_{a}(t)$ expanding with constant velocity $v_{e}>v_{a}$.

To study the principal kinetic quantities, it is convenient to introduce the notation $d=\delta t_{0}$. Then we consider the space-times with $s \neq 1$ for which we solve Eq. (41), finding the final time and redshift,

$$
\begin{aligned}
t_{f} & =t_{0}[1+\delta(1-s)]^{\frac{1}{1-s}}, \\
1+z & =a\left(t_{f}\right)=[1+\delta(1-s)]^{\frac{s}{1-s}} .
\end{aligned}
$$

The wave packet depends on geometry only through the variable (179) that now can be written for all the models with

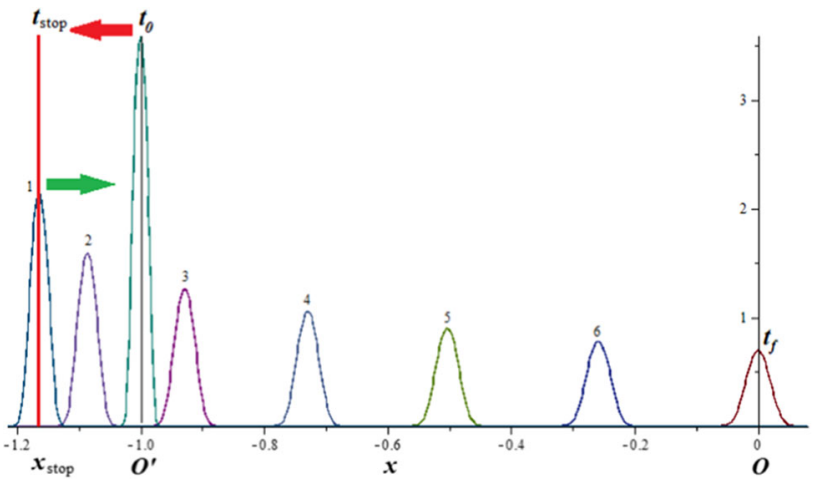

Fig. 3 Propagation of the wave packet with $\gamma=0.1$ and $\bar{k}=1000$ in the space-time with $s=0.3$ and $r_{a}\left(t_{0}\right)=\frac{1}{3}$. The packet is prepared in $O^{\prime}$ at the time $t_{0}=0.1$ and $d=1>r_{a}\left(t_{0}\right)$ and measured in $O$ at the time $t_{f}=1.9504$. We plot eight consecutive profiles of the maximum intensity $I(t, x)$ (in logarithmic scale) between $t_{0}$ and $t_{f}$ at equal time intervals, $\Delta t=0.2643$. The maximum intensity stops in $x_{\text {stop }}=-1.1642$ at the time $t_{\text {stop }}=0.3492$

$s \neq 1$ as

$$
\begin{aligned}
X(t, x) & =\int_{t_{0}}^{t} \frac{\mathrm{d} t}{a(t)}-\frac{x}{a(t)}-d \\
& =\left(\frac{t_{0}}{t}\right)^{s}\left(\frac{t}{1-s}-x\right)-\frac{t_{0}}{1-s}-d,
\end{aligned}
$$

bearing in mind that $-d \leq x \leq 0$ is the space coordinate of the geodesic $O^{\prime} O$ and $a\left(t_{0}\right)=1 \rightarrow d\left(t_{0}\right)=d$. For $X(t, x)=0$, we obtain the null geodesic of the maximum intensity

$x_{m}(t)=\frac{t}{1-s}-\left(\frac{t}{t_{0}}\right)^{s}\left(d+\frac{t_{0}}{1-s}\right)$,

which is singular in $s=1$.

The space-time with $s=1$ is called the Milne-type universe [34], since this is spatially flat, having sources as in Eq. (194), in contrast to the genuine Milne one which is flat, without sources, but with curved space sections [23]. In this case Eqs. (199) and (200) are undefined so that we must replay the procedure, finding

$t_{f}=t_{0} e^{\delta}, \quad 1+z=a\left(t_{f}\right)=e^{\delta}$,

and

$$
\begin{aligned}
X(t, x) & =t_{0}\left(\ln \frac{t}{t_{0}}-\frac{x}{t}-\delta\right), \\
x_{m}(t) & =t\left(\ln \frac{t}{t_{0}}-\delta\right) .
\end{aligned}
$$

We can verify that the functions of $s$ defined by Eqs. (197) and (198) are continuous in $s=1$, where their limits are just the values given by Eq. (201). Therefore, these functions are increasing monotonously with $s$ such that $t_{f}>t_{0}$ and $z>0$ for any $s>0$. The space-time with $s=0$ is just the Minkowski one for, which $t_{f}-t_{0}=d$ and $z=0$. Note that 


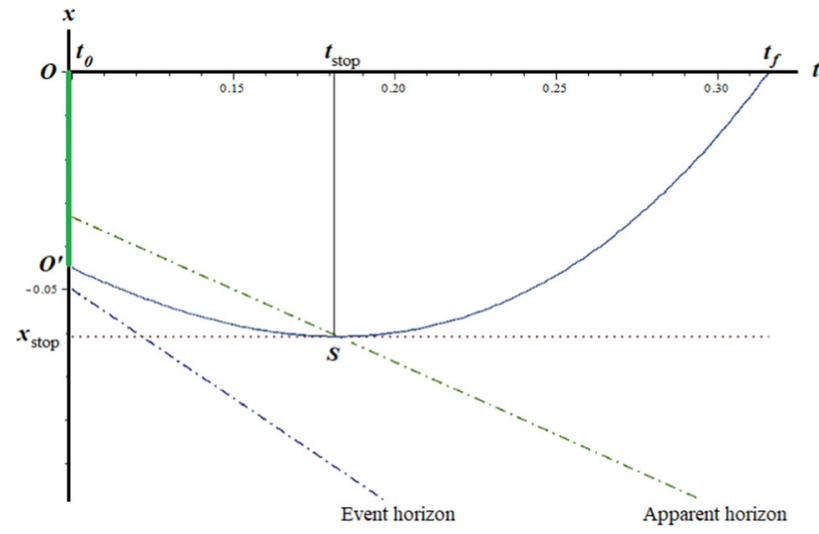

Fig. 4 Profile of the null geodesic $x_{m}(t)$ of the maximum intensity of the wave packet with $\gamma=0.001$ and $\bar{k}=10000$ in the space-time with $s=3, r_{a}\left(t_{0}\right)=0.0333$ and $r_{e}\left(t_{0}\right)=0.05$. The packet is prepared in $O^{\prime}$ at the time $t_{0}=0.1$ and $r_{a}\left(t_{0}\right)<d=0.045<r_{e}\left(t_{0}\right)$ and measured in $O$ at the time $t_{f}=0.3162$. The maximum intensity stops in $x_{\text {stop }}=-0.0608$ at the time $t_{\text {stop }}=0.1825$

our parameters must comply with the condition $0<t_{0}<t_{f}$ which determines the domain of the parameter $\delta$ as

$$
\begin{array}{cccc}
s<1: & 1+\delta(1-s)>1 & \rightarrow & \delta>0, \\
s=1: & e^{\delta}>1 & \rightarrow & \delta>0, \\
s>1: 0 \leq 1+\delta(1-s)<1 & \rightarrow & 0<\delta \leq \frac{1}{s-1},
\end{array}
$$

observing that the only restriction is given by the event horizons arising for $s>1$.

\subsection{Graphical analysis}

Now we may graphically analyse the time evolution of the intensity (178) that now reads

$I(t, x)=\left(\frac{t_{0}}{t}\right)^{4 s} I_{0}[X(t, x)]$,

where $I_{0}[X(t, x)]$ is the intensity (191) depending on the variable (199). We chose the parameters of the wave packet in order to obtain intuitive plottings in the whole space domain $-d \leq x \leq 0$ even though these parameters then remain far from their physical domains. For this reason we use arbitrary units, avoiding the quantitative analysis.

We focus first on the space-times with $s \leq 1$ in which the observer $O$ has only the apparent horizon (195). The observer $O^{\prime}$ may prepare the wave packet at the initial time $t_{0}$ either inside or outside this horizon. When this is inside, $d<r_{a}\left(t_{0}\right)$, then the one-dimensional wave packet that has to be measured by $O$ starts in $O^{\prime}$ with the initial velocity $0<$ $v_{m}\left(t_{0}\right)<1$ resulting from Eq. (45). The maximum intensity propagates accelerating straightforward to $O$ as in Fig. 1, having a severe decay $\left(\propto t^{-4 s}\right)$ and a moderate dispersion $\left(\propto t^{-s}\right)$. The initial velocity is less than the speed of light because of the background expansion dragging back the wave

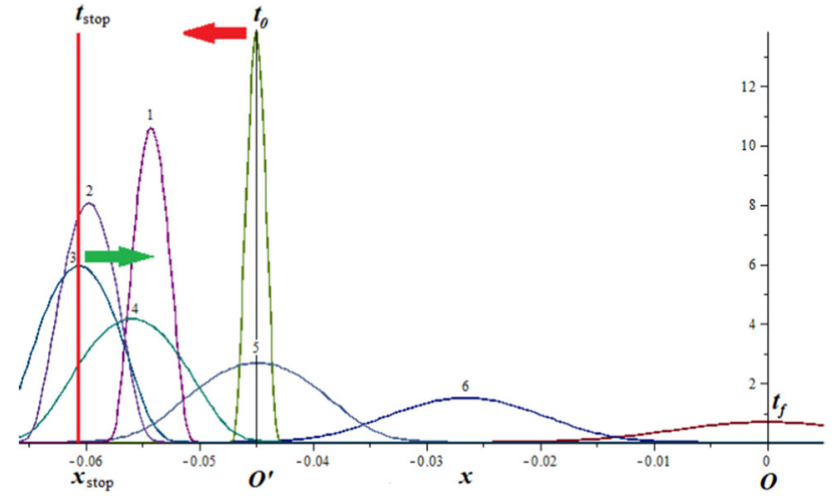

Fig. 5 Propagation of the wave packet with $\gamma=0.001$ and $\bar{k}=10000$ in the space-time with $s=3, r_{a}\left(t_{0}\right)=0.0333$ and $r_{e}\left(t_{0}\right)=0.05$. The packet is prepared in $O^{\prime}$ at the time $t_{0}=0.1$ and $r_{a}\left(t_{0}\right)<d=0.045<$ $r_{e}\left(t_{0}\right)$ and measured in $O$ at the time $t_{f}=0.3162$. We plot eight consecutive profiles of the maximum intensity $I(t, x)$ (in logarithmic scale) between $t_{0}$ and $t_{f}$ at equal time intervals, $\Delta t=0.0308$. The maximum intensity stops in $x_{\text {stop }}=-0.0608$ at the time $t_{\text {stop }}=0.1825$

packet. When the wave packet is prepared just on the apparent horizon, $d=r_{a}\left(t_{0}\right)$, then $v_{m}\left(t_{0}\right)=0$, the maximum intensity accelerating up to the speed of light detected by $O$.

More interesting is the case when $O^{\prime}$ prepares the packet outside the apparent horizon of $O$, in the initial point $x_{0}=$ $-d$ with $d>r_{a}\left(t_{0}\right)$. Then from Eq. (45) we see that the initial velocity, $v_{m}\left(t_{0}\right)<0$, is oriented in the backward direction because of a stronger dragging-back effect arising beyond the apparent horizon. To illustrate how the maximum intensity is propagating, we plot in Fig. 2 the function $x_{m}(t)$ of its null geodesic. The maximum intensity moves in the backward direction, being decelerated until this stops in the point $x_{\text {stop }}$ at the time $t_{\text {stop }}$ when the apparent horizon reaches the packet, $\left|x_{m}\left(t_{\text {stop }}\right)\right|=r_{a}\left(t_{\text {stop }}\right)$. This is a turning point, since for $t>t_{\text {stop }}$, the packet remains inside the apparent horizon, propagating accelerated in the forward direction, with decay and dispersion as in Fig. 3, such that it is detected in $O$ as having the speed of light.

In the space-times with $s>1$, the observer $O$ has an event horizon behind the apparent one such that we can test the above effect assuming that the observer $O^{\prime}$ prepares the packet outside the apparent horizon but inside the event one, $r_{a}\left(t_{0}\right)<d<r_{e}\left(t_{0}\right)$. The function $x_{m}(t)$ plotted in Fig. 4 lays out a turning point as in the previous case, determining a similar motion of the maximum intensity, as shown in Fig. 5. In other respects, we see that the presence of the event horizon does not affect the behaviour of the wave packet inside it. However, it would be interesting to see what happens when the packet is prepared on or beyond the event horizon, as we did in the case of the de Sitter space-time [13]. Unfortunately, this is impossible to achieve here because of some quantities that become complex-valued on and beyond the event horizon. 
We thus remain with only the effect of the apparent horizon in which the light seems to go back until it stops, turning then to the forward direction. This horizon effect, due to the spacetime expansion dragging back the wave packet, depends on the constant velocity $v_{a}=\frac{1}{s}$ of the apparent horizon. For $s<1$ this exceeds the speed of light, but for $s>1$ it is less than 1 . This explains why the deceleration period, $t_{\text {stop }}-t_{0}$, is much longer in the last case, as we can see comparing Figs. 3 and 5, where this interval represents 0.13 and 0.38 , respectively, from the total propagation time.

We must specify that this horizon effect cannot be observed by $O$ during the causal propagation of the photon whose parameters may be measured only in $x=0$ when this is detected. Nevertheless, the observer can deduce indirectly whether the packet is prepared inside or outside the apparent horizon measuring the redshift. If at the initial time the source was just on the apparent horizon, then Eq. (198) yields the measured redshift

$z_{a}=s^{\frac{s}{s-1}}-1$,

depending only on the geometry. Therefore, if $O$ measures a redshift $z<z_{a}$, then the source was inside the apparent horizon, while for $z>z_{a}$ it was outside.

The conclusion is that, measuring the redshift, the observer $O$ may deduce the decay of the maximum intensity $\propto$ $(1+z)^{-4}$, the dispersion $\propto(1+z)$ and the above horizon effect. Obviously, in the flat case when $z=0$, all these effects disappear.

\section{Concluding remarks}

We presented the complete classical and quantum theory of the Maxwell free field minimally coupled to the gravity of the spatially flat FLRW space-times. The results derived here are either generalizations of our previous ones we obtained for the de Sitter expanding universe $[10,12,13]$ or original results we present here for the first time. These are the role of the scaling (15) in the flat limit and canonical quantization, the electrodynamics in physical frames, the electromagnetic field generated by a system of static charges, the propagators in physical frames and the horizon effect.

According to our general method, we performed the calculations in conformal frames, transforming the results in the physical ones where these obtain a physical meaning. Thus we are faced with two different approaches, the familiar one in conformal frames where we take over the results of special relativity, and the new classical or quantum electrodynamics in physical frames, which seems to be a new and different world which deserves to be studied carefully.

Under such circumstances, a justified question is how we can reveal this new phenomenology in further experiments and observations. As mentioned, for studying the propaga- tion, it is enough to know the geometry and redshift for deducing the decay, dispersion and the relative position of the source with respect to the apparent horizon. The acceleration can be estimated theoretically but is never measurable, as the observer measures only the final velocity, which is just the speed of light.

The difficulties in acquiring empirical data arise when we look for other effects as, for example, that which we called here the geometric induction. Obviously, in our actual expanding universe, all the local experiments confirm the special relativistic classical or quantum electrodynamics which is independent of gravity. This suggests that the abovementioned effect or other similar ones may be of interest only in very strong gravitational fields such as those of the inflationary epoch. For this reason, we believe that this new electrodynamics must be developed in various cosmological scenarios, analysing different processes for finding relic effects that could be identified in the present astrophysical observations.

Data Availability Statement This manuscript has no associated data or the data will not be deposited. [Authors' comment: This paper is theoretical without using or producing data.].

Open Access This article is licensed under a Creative Commons Attribution 4.0 International License, which permits use, sharing, adaptation, distribution and reproduction in any medium or format, as long as you give appropriate credit to the original author(s) and the source, provide a link to the Creative Commons licence, and indicate if changes were made. The images or other third party material in this article are included in the article's Creative Commons licence, unless indicated otherwise in a credit line to the material. If material is not included in the article's Creative Commons licence and your intended use is not permitted by statutory regulation or exceeds the permitted use, you will need to obtain permission directly from the copyright holder. To view a copy of this licence, visit http://creativecomm ons.org/licenses/by/4.0/.

Funded by $\mathrm{SCOAP}^{3}$.

\section{References}

1. I.K. Tamm, J. Russ, Phys. Chem. Soc. Phys. Part 56, 248 (1924)

2. J. Plebanski, Phys. Rev. 118, 1396 (1960)

3. B. Mashhoon, Phys. Rev. D 8, 4297 (1973)

4. A. Higuchi, L.Y. Cheong, Phys. Rev. D 78, 084031 (2008)

5. A. Higuchi, L.Y. Cheong, J.R. Nicholas, Phys. Rev. D 80, 107502 (2009)

6. S. Faci, E. Huguet, J. Queva, J. Renaud, Phys. Rev. D 80, 124005 (2009)

7. S. Faci, E. Huguet, J. Renaud, Phys. Rev. D 84, 124050 (2011)

8. D. Bini, G. Esposito, R.V. Montaquila, GRG 42, 51 (2010)

9. A.A. Saharin, A.S. Kotanjian, H.A. Nersisyan, Phys. Lett. B 728, $141(2014)$

10. I.I. Cotăescu, C. Crucean, Prog. Theor. Phys. 124, 1 (2010)

11. I.I. Cotăescu, C. Crucean, Phys. Rev. D 87, 044016 (2013)

12. I.I. Cotăescu, Eur. Phys. J. C 81, 553 (2021)

13. I.I. Cotăescu, I. Cotăescu Jr., Eur. Phys. J. C 81, 667 (2021)

14. P. Painlevé, CR Acad. Sci. (Paris) 173, 677 (1921)

15. A. Messiah, Quantum Mechanics (Dover, New York, 1999) 
16. S. Drell, J.D. Bjorken, Relativistic Quantum Fields (Me Graw-Hill Book Co., New York, 1965)

17. K. Huang, Quantum Field Theory (Wiley, New York, 1989)

18. I.I. Cotăescu, Mod. Phys. Lett. A 22, 2965 (2007)

19. I.I. Cotăescu, C. Crucean, A. Pop, Int. J. Mod. Phys. A 23, 2563 (2008)

20. I.I. Cotăescu, Int. J. Mod. Phys. A 35, 2030019 (2020)

21. I.I. Cotăescu, Chin. Phys. C 45, 105101 (2021)

22. D. Bini, C. Cherubini, C. Chicone, B. Mashhoon, Class. Quantum Gravity 25, 225014 (2008)

23. N.D. Birrell, P.C.W. Davies, Quantum Fields in Curved Space (Cambridge Univ. Press, Cambridge, 1982)

24. G. Lemaître, J. Math. Phys. (Cambridge, Mass.) 4, 188 (1925)
25. I.I. Cotăescu, Mod. Phys. Lett. A 32, 1750223 (2017)

26. G.E. Lemaître, Ann. Soc. Sci. de Bruxelles 47A, 49 (1927)

27. G.E. Lemaître, MNRAS 91, 483 (1931)

28. E. Hubble, Proc. Natl. Acad. Sci. 15, 168 (1929)

29. E. Harrison, Astrophys. J. 403, 28 (1993)

30. W. Rindler, MNRAS 116, 662 (1956)

31. W. Rindler, Essential of Relativity (Springer, Heidelberg, 1969)

32. K. Bleuler, Helv. Phys. Acta (in German) 23, 567 (1950)

33. S. Gupta, Proc. Phys. Soc. 63A, 681 (1950)

34. I.I. Cotăescu, D. Popescu, Chin. Phys. C 44, 055104 (2020) 